https://helda.helsinki.fi

\title{
Large deviations and fluctuation theorem for the quantum heat current in the spin-boson model
}

\section{Aurell, Erik}

2020-05-15

Aurell , E , Donvil , B \& Mallick , K 2020 , ' Large deviations and fluctuation theorem for the quantum heat current in the spin-boson model ' , Physical Review E , vol. 101, no. 5 , 052116 . https://doi.org/10.1103/PhysRevE.101.052116

http://hdl.handle.net/10138/319671

https://doi.org/10.1103/PhysRevE.101.052116

unspecified

publishedVersion

Downloaded from Helda, University of Helsinki institutional repository.

This is an electronic reprint of the original article.

This reprint may differ from the original in pagination and typographic detail.

Please cite the original version. 


\title{
Large deviations and fluctuation theorem for the quantum heat current in the spin-boson model
}

\author{
Erik Aurell* \\ KTH Royal Institute of Technology, AlbaNova University Center, SE-106 91 Stockholm, Sweden \\ and Faculty of Physics, Astronomy and Applied Computer Science, Jagiellonian University, 30-348 Kraków, Poland \\ Brecht Donvil $\oplus^{\dagger}$ \\ Department of Mathematics and Statistics, University of Helsinki, P.O. Box 68, 00014 Helsinki, Finland \\ Kirone Mallick ${ }^{\ddagger}$ \\ Institut de Physique Théorique, Université Paris-Saclay, CEA and CNRS, 91191 Gif-sur-Yvette, France
}

(Received 13 November 2019; revised manuscript received 11 February 2020; accepted 16 March 2020; published 15 May 2020)

\begin{abstract}
We study the heat current flowing between two baths consisting of harmonic oscillators interacting with a qubit through a spin-boson coupling. An explicit expression for the generating function of the total heat flowing between the right and left baths is derived by evaluating the corresponding Feynman-Vernon path integral by performing the noninteracting blip approximation (NIBA). We recover the known expression, obtained by using the polaron transform. This generating function satisfies the Gallavotti-Cohen fluctuation theorem, both before and after performing the NIBA. We also verify that the heat conductance is proportional to the variance of the heat current, retrieving the well-known fluctuation dissipation relation. Finally, we present numerical results for the heat current.
\end{abstract}

DOI: 10.1103/PhysRevE.101.052116

\section{INTRODUCTION}

The flow of a nonvanishing macroscopic current of energy, charge, matter, or information that breaks time-reversal invariance is a fingerprint of nonequilibrium behavior. A paradigmatic model for such a situation consists of a small system, with a finite number of degrees of freedom, that connects two large reservoirs in different thermodynamic states. The ensuing stationary state can not be described by the standard laws of thermodynamics: in particular, the steady-state statistics are not given by a Gibbs ensemble. The theoretical analysis of simple models, whether classical or quantum, provides us with a wealth of information about far-from-equilibrium physics and has stimulated numerous studies in the last two decades [1-8].

Quantum systems based on nanoscale integrated circuits are very effective for the study of quantum phenomena and are good candidates for possible applications. This is due to their macroscopic size and the ensuing ability to manipulate them. For any application minimizing or controlling the heat flow is essential. Therefore there has been a great deal of experimental [9-16] and theoretical interest [17-20] in studying the heat flow in such circuits. The vast majority of theoretical studies have been focused on the weak coupling regime, for which well-controlled approximation schemes are available. For example, in the case of a small system interacting with an environment, it is possible to integrate out the bath from

\footnotetext{
*eaurell@kth.se

†brecht.donvil@helsinki.fi

${ }^{\ddagger}$ kirone.mallick@ipht.fr
}

the full dynamics and express the resulting system dynamics in terms of a Lindblad equation [3,4,21]. In this case, heat currents can be studied in terms of energy changes of the system. However, the weak coupling assumption deviates from exact treatments quantitatively and qualitatively already at moderately low couplings [22].

There have been various earlier studies in the strong coupling regime. Based on the polaron transform, the authors of Ref. [23] obtained an analytical expression for the heat current through an $N$ level system. The polaron transform provides a shortcut for the noninteracting blip approximation (NIBA) [24,25]. The full generating function for a spinboson system was derived, using the polaron transform, in Refs. [26,27] and reviewed in Ref. [28]. The authors of Refs. [29-31] derived a nonequilibrium polaron-transformed Redfield equation that unifies strong and weak coupling behavior. In Refs. [32-34] the authors start from the generating function of the heat current to study its first moment. Numerical studies include simulations based on hierarchical equations of motion [35-40] the quasiadiabatic propagator path integral (QuAPI) [41,42], the iterative full counting statistics path integral [43], the multiconfiguration time-dependent Hartree (MCTDH) approach [44], the stochastic Liouvillian algorithm [45], and other Monte Carlo approaches [46]. Other recent contributions are Refs. [15,47-52].

In this paper we consider a qubit coupled to two (or more) thermal baths. We derive the full generating function for the spin boson by directly applying the noninteracting blip approximation (NIBA), without passing through the polaron transform as was done in the original derivation [26,27]. We show that we recover the result by Refs. [26,27]. Following Ref. [34], this generating function can be written explicitly 
as Feynman-Vernon-type path integral. Relying on a modified version of the NIBA, an expression for the first moment of the generating function, i.e., the average heat current, was obtained by directly applying the NIBA in Ref. [34]. Furthermore, we discuss the Gallavotti-Cohen relation (see Refs. [7,53-57] and references therein), which holds before and after the NIBA, as derived in Refs. [26,27], in terms of an explicit time reversal. Finally, we find a fluctuation-dissipation relation between the variance of the heat current and the thermal conductance.

The paper is structured as follows. In Sec. II, we briefly introduce the spin-boson model that we will analyze. In Sec. III, the generating function of the heat current is calculated after performing the NIBA approximation. In Sec. IV we discuss the Gallavotti-Cohen relation before and after the NIBA. In Sec. V we invert the Laplace transform of the generating functions for small $\alpha$ and obtain a fluctuation-dissipation relation between the variance of the heat current and the heat conductance. Finally, in Sec. VI we numerically evaluate the first moment of the generating function. Technical details are provided in the Appendixes.

\section{MODEL}

The spin-boson model is a prototype for understanding quantum coherence in presence of dissipation [58-62]. It can be viewed as a variant of the Caldeira-Leggett model in which a quantum particle interacts with a bath of quantummechanical oscillators. In the spin-boson model, a two-level system modeled by a spin- $1 / 2$ degree of freedom is put in contact with one or more heat-baths. The literature in the subject is vast and we refer the reader to some reviews and to the references therein [2,62-64].

In this paper, we will study two baths made of harmonic oscillators that interact with a qubit via the spin-boson interaction. Although there is no direct interaction between the baths, energy will be transferred through the qubit. The Hamiltonian governing the total evolution of the qubit and of the baths is given by

$$
H=H_{S}+H_{L}+H_{R}+H_{L S}+H_{R S} .
$$

The qubit Hamiltonian is given by

$$
H_{S}=-\hbar \frac{\Delta}{2} \sigma_{x}+\frac{\epsilon}{2} \sigma_{z} .
$$

The left bath and right bath Hamiltonians are given by

$$
\begin{aligned}
H_{L} & =\sum_{b \in C} \frac{p_{b, L}^{2}}{2 m_{b, L}}+\frac{1}{2} m_{b, L} \omega_{b, L}^{2} q_{b, L}^{2} \\
H_{R} & =\sum_{b \in R} \frac{p_{b, R}^{2}}{2 m_{b, R}}+\frac{1}{2} m_{b, R} \omega_{b, R}^{2} q_{b, R}^{2} .
\end{aligned}
$$

Finally, the system-bath interactions are of the spin-boson type [62]

$$
\begin{aligned}
& H_{L S}=-\sigma_{z} \sum_{b \in L} C_{b, L} q_{b, L} \\
& H_{R S}=-\sigma_{z} \sum_{b \in H} C_{b, R} q_{b, R} .
\end{aligned}
$$

The effects of the environment are embodied in the spectral density of the environmental coupling [2] (one for each bath):

$$
J^{R / L}(\omega)=\sum_{b \in R / L} \frac{\left(C_{b}^{R / L}\right)^{2}}{2 m_{b} \omega_{b}} \delta\left(\omega-\omega_{b}\right) .
$$

We will assume a Ohmic spectrum with an exponential cut-off determined by the frequency $\Omega$

$$
J^{R / L}(\omega)=\frac{2}{\pi} \eta_{R / L} \omega \exp \left(-\frac{\omega}{\Omega}\right) .
$$

We denote by $U_{t}$ the unitary evolution operator of the total system and assume that the baths are initially at thermal equilibrium and are prepared in Gibbs states at different temperatures. For an initial state of the qubit $|i\rangle$ and a final state $|f\rangle$, the generating function of the heat current is defined as

$$
\begin{aligned}
G_{i, f}(\vec{\alpha}, t)= & \operatorname{tr}_{R, L}\langle f| e^{i\left(\alpha_{R} H_{R}+\alpha_{L} H_{L}\right) / \hbar} U_{t} e^{-i\left(\alpha_{R} H_{R}+\alpha_{L} H_{L}\right) / \hbar} \\
& \times\left(\rho_{\beta_{L}} \otimes \rho_{\beta_{R}} \otimes|i\rangle\langle i|\right) U_{t}^{\dagger}|f\rangle,
\end{aligned}
$$

with $\vec{\alpha}=\left(\alpha_{R}, \alpha_{L}\right)$. The trace is taken over all the degrees of freedom of the baths. This generating function will allow us to calculate all the moments of the heat current: for example, by taking the first derivative of $\alpha_{L}$ and setting $\vec{\alpha}$ to zero gives the change in expected energy of the cold bath

$$
-\left.i \hbar \partial_{\alpha_{L}} G_{i, f}(\vec{\alpha})\right|_{\vec{\alpha}=0}=\operatorname{tr}_{R, L}\left[H_{L} \rho(t)\right]-\operatorname{tr}\left[H_{L} \rho(0)\right]=\Delta E_{L} .
$$

\section{CALCULATION OF THE GENERATING FUNCTION}

The full generating function (9) was calculated in Refs. $[26,27]$ using the polaron transform. In this section we aim to perform this calculation by explicitly applying the NIBA to (9). The first step of the calculation is to rewrite the trace in Eq. (9) as a Feynman-Vernon-type path integral [2]. After integrating over the left and the right bath, see Appendix A, the expression for the generating function is given by [34]

$$
G_{i, f}(\vec{\alpha}, t)=\int_{i, f} d X d Y e^{\frac{i}{\hbar} S_{0}[X]-\frac{i}{\hbar} S_{0}[Y]} \mathcal{F}_{\vec{\alpha}}[X, Y],
$$

where $\mathcal{F}_{\vec{\alpha}}$ is the influence functional. The paths $X$ and $Y$ are the forward and backwards path of the qubit, they take values \pm 1 . The forward path $X$ corresponds to the forward evolution operator $U_{t}$ in (11), and $Y$ corresponds to $U_{t}^{\dagger}$. In the absence of interactions with the baths, the dynamics of the qubit are fully described by the free qubit action $S_{0}$

$$
S_{0}[X]=-\frac{\epsilon}{2} \int d t X(t)-i \log (i \Delta d t / 2) \int|d X(t)| .
$$

The integral $\int|d X(t)|$ counts the amount of jumps in the path. Thus, when the path $X$ makes $n$ jumps, the second term gives the weight $(i \Delta d t / 2)^{n}$. The effect of the influence functional is to generate interactions between the forward and backward paths; it also embodies the dependence on the parameters $\vec{\alpha}$.

$$
\mathcal{F}_{\vec{\alpha}}[X, Y]=e^{\frac{i}{\hbar}\left(S_{i, \alpha_{L}}^{C}[X, Y]+S_{i, \alpha_{R}}^{R}[X, Y]\right)} e^{-\frac{1}{\hbar}\left(S_{r, \alpha_{L}}^{C}[X, Y]+S_{r, \alpha_{R}}^{R}[X, Y]\right)},
$$


where the real part of the interaction action is given by [65]

$$
\begin{aligned}
S_{r, \alpha_{R / L}}^{R / L}[X, Y]= & \int_{t_{i}}^{t_{f}} d t \int_{t_{i}}^{t} d s\left(\left(X_{t} X_{s}+Y_{t} Y_{s}\right) k_{r}^{R / L}(t-s)\right. \\
& -X_{t} Y_{s} k_{r}^{R / L}\left(t-s+\alpha_{R / L}\right) \\
& \left.-X_{s} Y_{t} k_{r}^{R / L}\left(t-s-\alpha_{R / L}\right)\right)
\end{aligned}
$$

and the imaginary part is defined as

$$
\begin{aligned}
S_{i, \alpha_{R / L}}^{R / L}[X, Y]= & \int_{t_{i}}^{t_{f}} d t \int_{t_{i}}^{t} d s\left(\left(X_{t} X_{s}-Y_{t} Y_{s}\right) k_{i}^{R / L}(t-s)\right. \\
& +X_{t} Y_{s} k_{i}^{R / L}\left(t-s+\alpha_{R / L}\right) \\
& \left.-X_{s} Y_{t} k_{i}^{R / L}\left(t-s-\alpha_{R / L}\right)\right) .
\end{aligned}
$$

The kernels that appear in these expressions are

$$
k_{i}^{j}(t-s)=\sum_{b} \frac{\left(C_{b, j}^{j}\right)^{2}}{2 m_{b, j} \omega_{b, j}} \sin \left[\omega_{b, j}(t-s)\right]
$$

and

$$
k_{r}^{j}(t-s)=\sum_{b} \frac{\left(C_{b, j}^{j}\right)^{2}}{2 m_{b, j} \omega_{b, j}} \operatorname{coth}\left(\frac{\hbar \omega_{b, j} \beta_{j}}{2}\right) \sin \left[\omega_{b, j}(t-s)\right],
$$

for $j=R, L$. The integral of the bath degrees of freedom being performed, the generating function is given as the qubit path integral (11) over two binary paths. This remaining expression can not be calculated exactly; in the next section, we will evaluate the generating function by resorting to the noninteracting blip approximation (NIBA).

\section{Performing the NIBA}

Originally, the idea of the NIBA was proposed in Ref. [60], see also Ref. [62], to compute transition probabilities between states of the qubit: this corresponds to taking $\alpha=0$ in (9). The paths $X$ and $Y$ being binary, there are only two possibilities at a given time: either $X=Y$, this is a sojourn, or $X=-Y$, this is a blip. The NIBA approximation relies on two assumptions (explained in Ref. [62]):

(i) The typical blip-interval time $\Delta t_{B}$ is much shorter than the typical sojourn-interval time $\Delta t_{S}: \Delta t_{B} \ll \Delta t_{S}$.

(ii) Bath correlations decay over times much smaller than the typical sojourn interval $\Delta t_{S}$.

For an Ohmic spectrum (8), these assumptions are valid for two regimes: (i) for $\epsilon=0$ and weak coupling and (ii) for large damping and/or at high temperatures [2].

Under these assumptions, the only nonzero contributions to the time integrals in the interaction part of the action (14) and (15) are obtained when

(i) $t$ and $s$ are in the same blip interval;

(ii) $t$ and $s$ are in the same sojourn interval;

(iii) $t$ is in a sojourn and $s$ is an adjacent blip interval; blip.

(iv) $t$ and $s$ are both in sojourn intervals separated by one

Other terms cannot contribute since then $t$ and $s$ will be situated in intervals separated by at least one sojourn, which does give a contribution under assumption (ii).
The strategy to perform the NIBA is to break up the integrals (14) and (15) over the whole time interval into a sum of the surviving parts, which can be evaluated separately. In the present work, we extend the NIBA to include nonzero $\alpha$ (see also Ref. [34]), which leads to a time shift in some of the kernels in the action (14) and (15). In the framework of our approximation, we consider values of $\alpha_{R / L}$, such that $\alpha_{R / L} \ll \Delta t_{S}$. Following the same reasoning as for $\alpha_{R / L}=0$, it is clear that under said additional assumption, the same terms as before have a chance of being nonzero. In Appendix B, we explicitly calculate the five different surviving terms ${ }^{1}$ after the NIBA. The resulting expression for the generating function can be written in terms of a transfer matrix $M(\alpha, t)$ :

$$
\begin{aligned}
G_{\uparrow \uparrow}(\vec{\alpha}, t)+G_{\uparrow \downarrow}(\vec{\alpha}, t) \\
=\left(\begin{array}{ll}
1 & 1
\end{array}\right) \sum_{n=0}^{+\infty}(-1)^{n}\left(\frac{\Delta}{2}\right)^{2 n} \int d t_{1} \ldots d t_{2 n} \mathbf{M}\left(\alpha, \Delta t_{2 n}\right) \\
\quad \times \mathbf{M}\left(\alpha, \Delta t_{2 n-2}\right) \ldots \mathbf{M}\left(\alpha, \Delta t_{2}\right)\left(\begin{array}{l}
1 \\
0
\end{array}\right)
\end{aligned}
$$

where $\Delta t_{2 j}=t_{2 j}-t_{2 j-1}$. The transfer matrix $\mathbf{M}$ is given by

$$
\mathbf{M}(\vec{\alpha}, t)=2\left(\begin{array}{cc}
A(t) & -B(\vec{\alpha}, t) \\
-C(\vec{\alpha}, t) & D(t)
\end{array}\right)
$$

Note that only the off-diagonal elements of the transfer matrix depend on $\alpha$. The functions $A, B, C$, and $D$ that appear as matrix elements in $\mathbf{M}$ are determined once the NIBA has been performed. Their values are given by

$$
\begin{aligned}
A(t)= & \cos \frac{1}{\hbar}\left[Z_{L}^{+}(t)+Z_{R}^{+}(t)-\epsilon t\right] e^{-\frac{1}{\hbar}\left[\Gamma_{L}^{+}(t)+\Gamma_{R}^{+}(t)\right]} \\
B(\vec{\alpha}, t)= & e^{-\frac{1}{\hbar}\left[\Gamma_{L}^{-}\left(\alpha_{L}, t\right)+\Gamma_{R}^{-}\left(\alpha_{R}, t\right)+2 i\left(R_{L}\left(\alpha_{L}, t\right)+R_{R}\left(\alpha_{R}, t\right)\right]\right.} \\
& \times \cos \frac{1}{\hbar}\left[Z_{L}^{-}\left(\alpha_{L}, t\right)+2 i F_{L}\left(\alpha_{L}, t\right)+Z_{R}^{-}\left(\alpha_{L}, t\right)\right. \\
& \left.+2 i F_{R}\left(\alpha_{L}, t\right)+\epsilon t\right] \\
C(\vec{\alpha}, t)= & e^{-\frac{1}{\hbar}\left[\Gamma_{L}^{-}\left(\alpha_{L}, t\right)+\Gamma_{R}^{-}\left(\alpha_{R}, t\right)+2 i\left(R_{L}\left(\alpha_{L}, t\right)+R_{R}\left(\alpha_{R}, t\right)\right]\right.} \\
& \times \cos \frac{1}{\hbar}\left[Z_{L}^{-}\left(\alpha_{L}, t\right)+2 i F_{L}\left(\alpha_{L}, t\right)+Z_{R}^{-}\left(\alpha_{L}, t\right)\right. \\
& \left.+2 i F_{R}\left(\alpha_{L}, t\right)-\epsilon t\right] \\
D(t)= & \cos \frac{1}{\hbar}\left[Z_{L}^{+}(t)+Z_{R}^{+}(t)+\epsilon t\right] e^{-\frac{1}{\hbar}\left[\Gamma_{L}^{+}(t)+\Gamma_{R}^{+}(t)\right]}
\end{aligned}
$$

All the auxiliary functions $Z_{j}^{ \pm}, \Gamma_{j}^{ \pm}, R_{j}$, and $F_{j}$, where the index $j=L, R$ refers to the left or the right bath, are determined in Appendix B. Assuming a Ohmic spectral density with exponential cutoff with frequency $\Omega(7)$, the explicit expressions of these functions are given in the following equations:

$$
Z_{j}^{+}(t)=\frac{2 \eta_{j}}{\pi} \int_{0}^{\infty} d \omega \frac{\sin (\omega t)}{\omega} e^{-\omega / \Omega}
$$

${ }^{1}$ For $\mathrm{c}$ the blip can be before or after the sojourn. These give different contributions and are calculated separately. Hence there are five different surviving terms. 


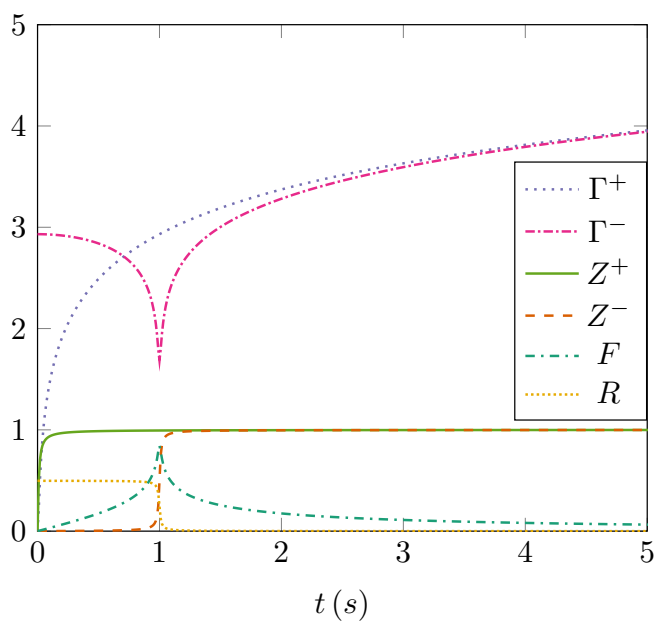

FIG. 1. Behavior of the functions (22) appearing in the definitions of the matrix elements (20) for $\alpha=1, \eta=1$ and $\beta=$ $\left(0.1 \mathrm{~K}_{B}\right)^{-1}$.

$$
\begin{aligned}
Z_{j}^{-}\left(\alpha_{j}, t\right) & =\frac{2 \eta_{j}}{\pi} \int_{0}^{\infty} d \omega \frac{\sin (\omega t)}{\omega} \cos \left(\omega \alpha_{j}\right) e^{-\omega / \Omega} \\
\Gamma_{j}^{+}(t) & =\frac{2 \eta_{j}}{\pi} \int_{0}^{\infty} d \omega \frac{1-\cos (\omega t)}{\omega} \operatorname{coth}\left(\frac{\omega \hbar \beta_{j}}{2}\right) e^{-\omega / \Omega} \\
\Gamma_{j}^{-}\left(\alpha_{j}, t\right)= & \frac{2 \eta_{j}}{\pi} \int_{0}^{\infty} d \omega\left(\frac{1-\cos (\omega t) \cos \left(\omega \alpha_{j}\right)}{\omega}\right. \\
R_{j}\left(\alpha_{j}, t\right)= & \frac{\eta_{j}}{\pi} \int_{0}^{\infty} d \omega \frac{\sin \left(\omega \alpha_{j}\right)}{\omega} \cos (\omega t) e^{-\omega / \Omega} \\
F_{j}\left(\alpha_{j}, t\right)= & \frac{\eta_{j}}{\pi} \int_{0}^{\infty} d \omega \frac{\operatorname{coth}\left(\frac{\omega \hbar \beta_{j}}{2}\right)}{\omega} \sin (\omega t) \sin \left(\omega \alpha_{j}\right) e^{-\omega / \Omega} .
\end{aligned}
$$

The behavior of these functions is shown in Fig. 1.

We will denote by $\tilde{\phi}$ the Laplace transform of a function $\phi(\vec{\alpha}, t)$, defined as follows:

$$
\tilde{\phi}(\vec{\alpha}, \lambda)=\int_{0}^{\infty} d t e^{-\lambda t} \phi(\vec{\alpha}, t) .
$$

Then, taking the Laplace transform of (18) leads us to

$$
\begin{aligned}
& \tilde{G}_{\uparrow \uparrow}(\vec{\alpha}, \lambda)+\tilde{G}_{\uparrow \downarrow}(\vec{\alpha}, \lambda) \\
& =\lambda^{-1}\left(\begin{array}{ll}
1 & 1
\end{array}\right)\left(\sum_{n=0}^{+\infty}(-1)^{n}\left(\frac{\Delta}{2}\right)^{2 n} \lambda^{-n} \tilde{\mathbf{M}}^{n}(\alpha, \lambda)\right)\left(\begin{array}{l}
1 \\
0
\end{array}\right) .
\end{aligned}
$$

We call $\lambda_{+}(\vec{\alpha}, \lambda)$ and $\lambda_{-}(\vec{\alpha}, \lambda)$ the eigenvalues of the $2 \times 2$ matrix $\tilde{\mathbf{M}}(\vec{\alpha}, \lambda)$, with corresponding left eigenvectors $v_{+}(\vec{\alpha}, \lambda)$ and $v_{-}(\vec{\alpha}, \lambda)$ and right eigenvectors $w_{+}(\vec{\alpha}, \lambda)$ and $w_{-}(\vec{\alpha}, \lambda)$. We can write $\tilde{\mathbf{M}}^{n}$ in terms of it eigenvectors and eigenvalues as

$$
\tilde{\mathbf{M}}^{n}=\lambda_{+}^{n}(\vec{\alpha}, t) w_{+}(\vec{\alpha}, t) v_{+}^{T}(\vec{\alpha}, t)+\lambda_{-}^{n}(\vec{\alpha}, t) w_{-}(\vec{\alpha}, t) v_{-}^{T}(\vec{\alpha}, t) .
$$

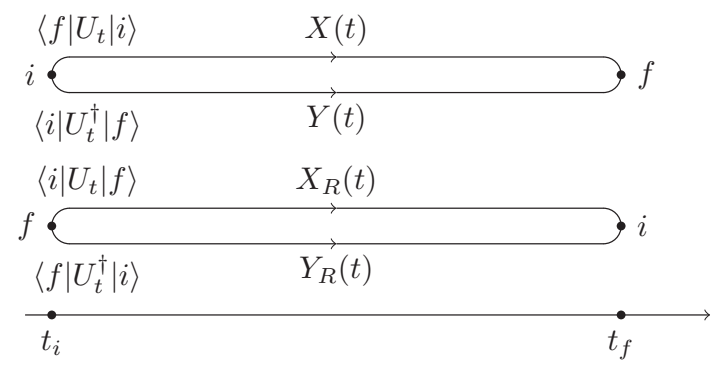

FIG. 2. Illustration of the (top) forward spin-state paths and the (bottom) time-reversed paths.

We have

$$
\begin{aligned}
\lambda_{ \pm}(\vec{\alpha}, \lambda)= & \tilde{A}(\lambda)+\tilde{D}(\lambda) \\
& \pm \sqrt{\left(\tilde{A}(\lambda)-\tilde{D}(\lambda)^{2}+4 \tilde{B}(\vec{\alpha}, \lambda) \tilde{C}(\vec{\alpha}, \lambda)\right.}
\end{aligned}
$$

Finally, the Laplace transform of the generating function takes a simpler form in the eigenbasis of $\tilde{\mathbf{M}}$ :

$$
\begin{aligned}
& \tilde{G}_{\uparrow \uparrow}(\vec{\alpha}, \lambda)+\tilde{G}_{\uparrow \downarrow}(\vec{\alpha}, \lambda) \\
& =\frac{Q_{+}(\vec{\alpha}, \lambda)}{\lambda+\left(\frac{\Delta}{2}\right)^{2} \lambda_{+}(\vec{\alpha}, \lambda)}+\frac{Q_{-}(\vec{\alpha}, \lambda)}{\lambda+\left(\frac{\Delta}{2}\right)^{2} \lambda_{-}(\vec{\alpha}, \lambda)},
\end{aligned}
$$

where we defined the amplitudes

$$
Q_{ \pm}=\left(\begin{array}{ll}
1 & 1
\end{array}\right) w_{ \pm} v_{ \pm}^{T}\left(\begin{array}{l}
1 \\
0
\end{array}\right)
$$

Using the relations

$$
\begin{aligned}
& 2 R_{j}\left(\alpha_{j}, t\right) \pm Z^{-}\left(\alpha_{j}, t\right)=Z^{+}\left(\alpha_{j} \pm t\right) \\
& 2 F_{j}\left(\alpha_{j}, t\right) \pm \Gamma^{-}\left(\alpha_{j}, t\right)=\Gamma^{+}\left(\alpha_{j} \mp t\right)
\end{aligned}
$$

one can check that Eq. (28) recovers the result derived by Refs. [26,27].

\section{FLUCTUATION THEOREM PRE- AND POST-NIBA}

The authors of Ref. [26] proved a fluctuation relation for the generating function after the NIBA was performed. They showed that the leading eigenvalue of the transfer matrix $\tilde{\mathbf{M}}$ is invariant under $\vec{\alpha} \rightarrow i\left(\beta_{L}, \beta_{R}\right)-\vec{\alpha}$. Here we show said fluctuation relation directly on $\mathbf{M}$ by considering a proper time reversal.

\section{A. Time reversal}

There are multiple ways to define a time reversal for a stochastic process, see Ref. [66]. In this work, we define a reversal for the qubit state paths $X(t)$ and $Y(t)$ as

$$
\begin{aligned}
X_{R}(t) & =Y\left(t_{f}+t_{i}-t\right) \\
Y_{R}(t) & =X\left(t_{f}+t_{i}-t\right),
\end{aligned}
$$

see Fig. 2. In the time-reversed path the forward and backward path interchange and run from $t_{f}$ to $t_{i}$. To illustrate this time reversal, let us note that for $\vec{\alpha}=0$, the generating function (9) can be written as

$$
G_{i, f}(0)=\operatorname{tr}_{R, C}\left(\rho_{\beta_{L}} \otimes \rho_{\beta_{R}}\langle i|U| f\rangle\left\langle f\left|U^{\dagger}\right| i\right\rangle\right) .
$$


Expressing the trace as a path integral and computing the trace over the bath variables gives

$$
G_{i, f}(\overrightarrow{0})=\int_{i, f} d X d Y e^{-\frac{i}{\hbar} S_{0}[X]+\frac{i}{\hbar} S_{0}[Y]} \mathcal{F}_{R}[X, Y]
$$

with influence functional

$$
\mathcal{F}_{R}[X, Y]=e^{\frac{i}{\hbar}\left(S_{i, R}^{L}+S_{i, R}^{R}\right)[X, Y]-\frac{1}{\hbar}\left(S_{r, R}^{L}+S_{r, R}^{R}\right)[X, Y]},
$$

where we defined the real part of the action as

$$
\begin{aligned}
S_{r, R}^{R / L}[X, Y]= & \int_{t_{i}}^{t_{f}} d t \int_{t_{i}}^{t} d s\left(\left(X_{t} X_{s}+Y_{t} Y_{s}\right) k_{r}^{R / L}(t-s)\right. \\
& \left.-X_{t} Y_{s} k_{r}^{R / L}(t-s)-X_{s} Y_{t} k_{r}^{R / L}(t-s)\right)
\end{aligned}
$$

and the imaginary part

$$
\begin{aligned}
S_{i, R}^{R / L}[X, Y]= & \int_{t_{i}}^{t_{f}} d t \int_{t_{i}}^{t} d s\left(\left(Y_{t} Y_{s}-X_{t} X_{s}\right) k_{i}^{R / L}(t-s)\right. \\
& \left.+X_{t} Y_{s} k_{i}^{R / L}(t-s)-X_{s} Y_{t} k_{i}^{R / L}(t-s)\right) .
\end{aligned}
$$

Now taking $X(t) \rightarrow X_{R}(t)$ and $Y(t) \rightarrow Y_{R}(t)$, retrieves the expression for the generating function (11) for $\vec{\alpha}=0$.

\section{B. Gallavotti-Cohen symmetry}

Let us define the time-reversed generating function as

$$
\begin{aligned}
G_{f i}^{R}\left(\alpha_{R}, \alpha_{L}, t\right)= & \operatorname{tr}_{R, L}\langle i| e^{i\left(\alpha_{R} H_{R}+\alpha_{L} H_{L}\right) / \hbar} U_{t}^{\dagger} e^{-i\left(\alpha_{R} H_{R}+\alpha_{L} H_{L}\right) / \hbar} \\
& \times\left(\rho_{\beta_{L}} \otimes \rho_{\beta_{R}} \otimes|f\rangle\langle f|\right) U_{t}|i\rangle
\end{aligned}
$$

Before performing the NIBA, it is straightforward to show from the definition of the generating function (11) and (37) that the Gallavotti-Cohen relation holds:

$$
G_{i f}\left(i \beta_{R} \hbar-\alpha_{R}, i \beta_{L} \hbar-\alpha_{L}, t\right)=G_{f i}^{R}\left(\alpha_{R}, \alpha_{L}, t\right),
$$

see Ref. [57] for a detailed discussion on the Gallavotti-Cohen relation for interacting systems. After integrating out the bath, the above equation can be checked using the time reversal defined in (31).

It is possible to show that the Gallavotti-Cohen relation (38) still holds after performing the NIBA. In order to do so we perform the NIBA on the time-reversed generating function $G_{f i}^{R}\left(\alpha_{R}, \alpha_{L}, t\right)$ following the same procedure as outlined in Sec. III. The result is of the form (18), with transfer matrix

$$
\overline{\mathbf{M}}(\vec{\alpha}, t)=2\left(\begin{array}{cc}
D(t) & -C(\vec{\alpha}, t) \\
-B(\vec{\alpha}, t) & A(t)
\end{array}\right)
$$

On the other hand, one can calculate that

$$
\mathbf{M}\left(i \hbar\left(\beta_{R}, \beta_{L}\right)-\vec{\alpha}, t\right)=2\left(\begin{array}{cc}
A(t) & -C(\vec{\alpha}, t) \\
-B(\vec{\alpha}, t) & D(t)
\end{array}\right) .
$$

Note that in the time reversal (31), we interchange the meaning of $X$ and $Y$, as illustrated in Fig. 2. Interchanging the roles of $X$ and $Y$ means flipping the diagonal elements in transfer matrix. Thus $\mathbf{M}\left[i \hbar\left(\beta_{R}, \beta_{L}\right)-\vec{\alpha}, t\right]$ and $\overline{\mathbf{M}}(\vec{\alpha}, t)$ are equivalent, proving that the Gallavotti-Cohen relation remains true after the performing the NIBA, as was shown by Ref. [26].

\section{FLUCTUATION-DISSIPATION RELATION}

In this section we aim to calculate the first and second moment of the heat current in the steady state. In steady state we only need to focus on one bath as the magnitude of the heat current is the same for both baths. Therefore, let us set $\alpha_{R}=0$ and write $\alpha=\alpha_{L}$. We invert the Laplace transform of the generating function up to second order in $\alpha$. This allows us direct access to the first and second moment of the heat current. In order to be self-contained, in Appendix C we present a derivation of the thermal conductance $\kappa(\mathrm{C} 16)$, which will appear in the fluctuation-dissipation relation.

Concretely, we look for poles of Eq. (28), by constructing a function

$$
\lambda(\alpha)=\lambda_{0}+\lambda_{1} \alpha+\lambda_{2} \alpha^{2}+O\left(\alpha^{3}\right),
$$

which solves

$$
\lambda(\alpha)+\left(\frac{\Delta}{2}\right)^{2} \lambda_{-}(\alpha, \lambda(\alpha))=0
$$

at all orders in $\alpha$. Hence for small $\alpha$, we have, in the long time limit

$$
\begin{aligned}
G_{i, f}(\alpha, t) & =\operatorname{Res}\left(\frac{e^{\lambda t} Q_{-}(\alpha, \lambda)}{\lambda(\alpha)+\left(\frac{\Delta}{2}\right)^{2} \lambda_{-}(\alpha, \lambda)}, \lambda(\alpha)\right) \\
& =\frac{e^{\lambda(\alpha) t} Q_{-}[\alpha, \lambda(\alpha)]}{1+\left(\frac{\Delta}{2}\right)^{2} \dot{\lambda}_{-}[\alpha, \lambda(\alpha)]} .
\end{aligned}
$$

(Note that in the large time limit the contribution of the $\lambda_{+}$is exponentially subdominant). Keeping in mind that $\lambda_{-}(0, \lambda)=$ 0 , the zeroth order of Eq. (42) gives.

$$
\lambda_{0}=0 \text {. }
$$

Equation (42) to the first order in $\alpha$ translates to

$$
\lambda_{1}+\left(\frac{\Delta}{2}\right)^{2} \lambda_{-}^{\prime}\left(0, \lambda_{0}\right)+\left(\frac{\Delta}{2}\right)^{2} \dot{\lambda}_{-}\left(0, \lambda_{0}\right) \lambda_{1},
$$

where the accent denotes the derivative to the first variable and a dot to the second. The steady-state heat current is given by $-i \hbar \lambda_{1}$. After some algebra we find that

$$
\lambda_{1}=i\left(\frac{\Delta}{2}\right)^{2} \frac{p_{+} \pi_{\downarrow}+p_{-} \pi_{\uparrow}}{\hbar\left(p_{+}+p_{-}\right)},
$$

where we defined

$$
\begin{aligned}
& C_{L}(t)=e^{-\frac{1}{\hbar} \Gamma_{L}^{+}(t)+\frac{i}{\hbar} Z_{L}^{+}(t)} \\
& C_{R}(t)=e^{-\frac{1}{\hbar} \Gamma_{R}^{+}(t)+\frac{i}{\hbar} Z_{R}^{+}(t)}
\end{aligned}
$$

and $\hat{C}_{L}(\omega), \hat{C}_{R}(\omega)$ their Fourier transforms. The fractions $p_{ \pm} /\left(p_{+}+p_{-}\right)$give the steady-state population for the qubit in the up or down state, with

$$
\begin{aligned}
& p_{+}=\int_{-\infty}^{\infty} d t C_{L}(t) C_{R}(t) e^{i \epsilon t} \\
& p_{-}=\int_{-\infty}^{\infty} d t C_{L}(t) C_{R}(t) e^{-i \epsilon t},
\end{aligned}
$$

and the power emitted from the up $\pi_{\downarrow}$ and down state $\pi_{\uparrow}$

$$
\begin{aligned}
& \pi_{\uparrow}=\frac{\hbar}{2 \pi} \int_{-\infty}^{\infty} d \omega \omega \hat{C}_{L}(\omega) \hat{C}_{R}(\epsilon-\omega) \\
& \pi_{\downarrow}=\frac{\hbar}{2 \pi} \int_{-\infty}^{\infty} d \omega \omega \hat{C}_{L}(\omega) \hat{C}_{R}(-\epsilon-\omega) .
\end{aligned}
$$


The convolution in the first line can be interpreted as the sum over qubit relaxation rates with energy $\omega$ going to the left bath and $-\omega+\epsilon$ to the right bath, and the second line similarly in terms of a qubit excitation [26]. Additionally, we define

$$
\begin{aligned}
& \Sigma^{+}=\frac{\hbar^{2}}{2 \pi} \int_{-\infty}^{\infty} d \omega \omega^{2} \hat{C}_{L}(\omega) \hat{C}_{R}(\epsilon-\omega) \\
& \Sigma^{-}=\frac{\hbar^{2}}{2 \pi} \int_{-\infty}^{\infty} d \omega \omega^{2} \hat{C}_{L}(\omega) \hat{C}_{R}(-\epsilon-\omega) .
\end{aligned}
$$

Similarly, an expression can be obtained for $\lambda_{2}$. In equilibrium, when $\beta_{R}=\beta_{C}$,

$$
\lambda_{2}=-\frac{\Delta^{2}}{4 \hbar^{2}} \frac{p_{-} \Sigma^{+}+p_{+} \Sigma^{-}+4 \pi_{\uparrow} \pi_{\downarrow}}{p_{+}+p_{-}} .
$$

Writing the explicit expression for $Q_{-}[\alpha, \lambda(\alpha)]$, straightforward algebra shows that

$$
Q_{-}[\alpha, \lambda(\alpha)]=1+O\left(\alpha^{3}\right)
$$

and we obtain that the generating function is given by

$$
\begin{aligned}
G_{i, f}(\alpha)= & e^{\left(\lambda_{1} \alpha+\lambda_{2} \alpha^{2}+O\left(\alpha^{3}\right)\right) t}\left\{1-\left(\frac{\Delta}{2}\right)^{2}\right. \\
& \times\left[\dot{\lambda}_{-}^{\prime}(0,0)+\lambda_{-}^{\prime \prime}(0,0) \lambda_{1}\right] \alpha \\
& +\left(\left(\frac{\Delta}{2}\right)^{2} \dot{\lambda}_{-}^{\prime}(0,0)\right)^{2} \alpha^{2} \\
& \left.-\left(\frac{\Delta}{2}\right)^{2} \dot{\lambda}_{-}^{\prime \prime}(0,0) \alpha^{2}+O\left(\alpha^{3}\right)\right\} .
\end{aligned}
$$

The first moment of the heat current is

$$
\langle\Delta E\rangle=-i \hbar t \lambda_{1}
$$

which correctly leads to the heat current defined in (C3). The variance of the heat current is then given by

$$
\begin{aligned}
\operatorname{Var}[\Delta E]= & -\hbar^{2} t\left\{2 \lambda_{2}-2\left[\dot{\lambda}_{-}^{\prime}(0,0)\right.\right. \\
& \left.\left.\left.+\lambda_{-}^{\prime \prime}(0,0) \lambda_{1}\right)\right] \lambda_{1}\right\}+O(t) .
\end{aligned}
$$

In equilibrium, $\lambda_{1}=0$, we find that

$$
\begin{aligned}
\lim _{t \rightarrow \infty} \frac{1}{t} \operatorname{Var}[\Delta E]=-2 \hbar^{2} \lambda_{2} \\
\quad=\frac{\Delta^{2}}{2} \frac{p_{-} \Sigma^{+}+p_{+} \Sigma^{-}+4 \pi_{\uparrow} \pi_{\downarrow}}{p_{+}+p_{-}} .
\end{aligned}
$$

Comparing to (C16), we find the following identity:

$$
\lim _{t \rightarrow \infty} \frac{1}{t} \operatorname{Var}[\Delta E]=2 \kappa,
$$

which proves the fluctuation-dissipation relation.

\section{NUMERICAL EVALUATION OF THE GENERATING FUNCTION}

In this section we numerically study the heat current predicted by (18), earlier numerical studies on the spin-boson model include, e.g., Refs. [23,26-31,67,68].

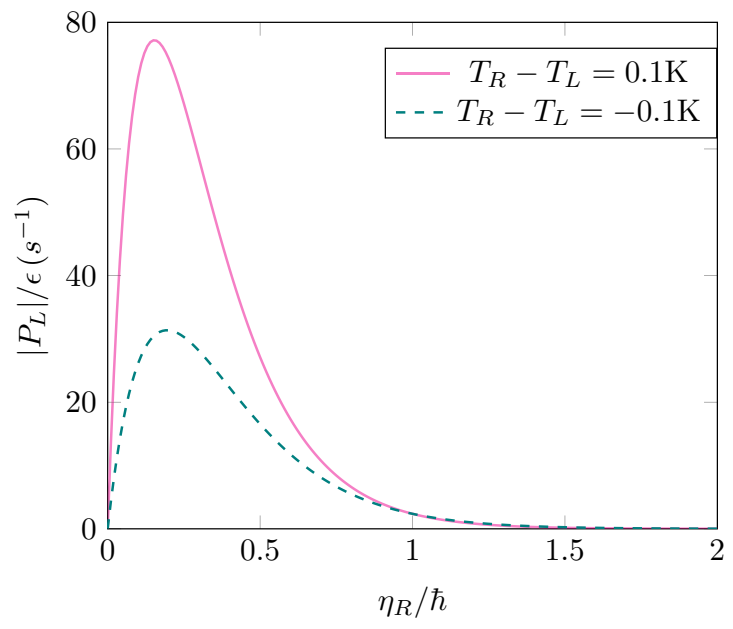

FIG. 3. Numerical evaluation of the heat current (C1). The purple lines are for $T_{R}=0.2 \mathrm{~K}, T_{L}=0.1 \mathrm{~K}$ and the blue lines for $T_{R}=$ $0.1 \mathrm{~K}, T_{L}=0.2 \mathrm{~K}$. The other parameters in the model are $\epsilon=1 \mathrm{~K} \times$ $k_{B}, \hbar \Delta=0.01 \epsilon, \Omega=100 \epsilon / \hbar$, and $\eta_{L}=\hbar$.

The heat current (C3) is completely determined by the functions $Z_{L / R}^{+}(t)$ and $\Gamma_{L / R}^{+}(t)$, defined in (22) and (21). For the Ohmic spectral density $J(\omega)$ with exponential cutoff (8), these functions have analytic solutions [62]

$$
\begin{gathered}
Z_{j}^{+}(t)=\eta_{j} \tan ^{-1}(\Omega t) \\
\Gamma_{j}^{+}(t)=\frac{1}{2} \eta_{j} \log \left(1+\Omega^{2} t^{2}\right)+\eta_{j} \log \left(\frac{\hbar \beta_{j}}{\pi t} \sinh \frac{\pi t}{\hbar \beta_{j}}\right),
\end{gathered}
$$

with $j=L, R$.

For our numerical analysis we consider the parameters $\epsilon=$ $1 \mathrm{~K} \times k_{B}, \hbar \Delta=0.01 \epsilon$, and $\Omega=100 \epsilon / \hbar$. Figure 3 shows the absolute value of the heat current to the left bath for a positive temperature gradient $\Delta T=T_{R}-T_{L}=0.1 \mathrm{~K}$ (full line) and for a negative gradient $-0.1 \mathrm{~K}$ (dashed line) in function of the coupling strength $\eta_{R}$, with $\eta_{L}=\hbar$ constant. The curves show rectification of the heat current, as was already observed by Refs. [23,67]: the current changes direction when the temperatures of the bath are exchanged, but the magnitudes are not equal.

Let $P_{L}$ be the power to the left bath and $P_{L}^{R}$ be the power to the left bath as the temperatures of the baths are exchanged. To quantify the amount of rectification, we define the rectification index as [16]

$$
R=\frac{\max \left(\left|P_{L}\right|,\left|P_{L}^{R}\right|\right)}{\min \left(\left|P_{L}\right|,\left|P_{L}^{R}\right|\right)} .
$$

The rectification index is shown in Fig. 4 for different range of temperatures of the right bath in function of the coupling parameter $\eta_{R}$. Larger temperature gradients lead to higher rectification.

The influence of a third bath, with temperature $T_{E}$, weakly coupled to the qubit on the rectification index $R$ is shown in Fig. 5. The left bath has constant coupling $\eta_{L}=\hbar$, the third bath has coupling $\eta_{E}=0.1 \hbar$ and the coupling of the right bath ranges from $0 \hbar$ to $1.5 \hbar$. The presence to the third bath leads to $P_{R} \neq-P_{L}$, which causes changes in the behavior 


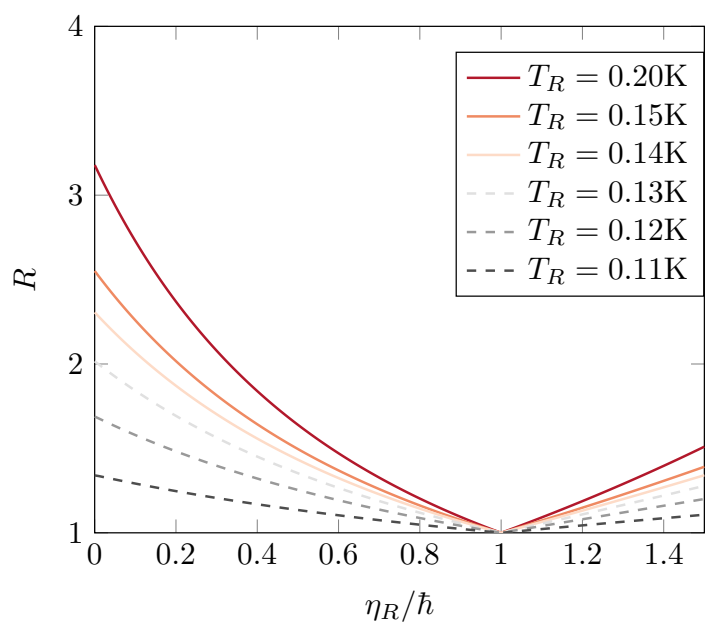

FIG. 4. Rectification index $R$, as defined in (60), for different values of $T_{R}$ and $T_{L}=0.1 \mathrm{~K}$. The other parameters are given in the caption of Fig. 3.

of the rectification index $R$. The black (full) line in Fig. 5 displays the rectification index without the third bath, the other curves show the rectification under the influence of the third bath. There are two clear qualitative deviations from the two-bath situation. First, the rectification no longer reaches a minimum at $\eta_{R}=1$, the minima are shifted to other values of $\eta_{R}$ and even additional minima appear. Second, divergences occur when the presence of the third bath leads to $P_{L}=0$ and $P_{L}^{R} \neq 0$, or the other way around. For example, at $\eta_{R}=0$ and $T_{E}=0.1 \mathrm{~K}$ the power $P_{L}=0$, since $T_{E}=T_{L}$ and there is no interaction with the right bath. When the temperatures are reversed, $T_{E} \neq T_{L}^{R}=T_{R}$ leading to $P_{L}^{R} \neq 0$. Theoretical studies of electronic systems have shown similar effect on the rectification due the influence of a third bath $[69,70]$, earlier numerical studies for the three-bath model in the spin-boson case are in Ref. [68].

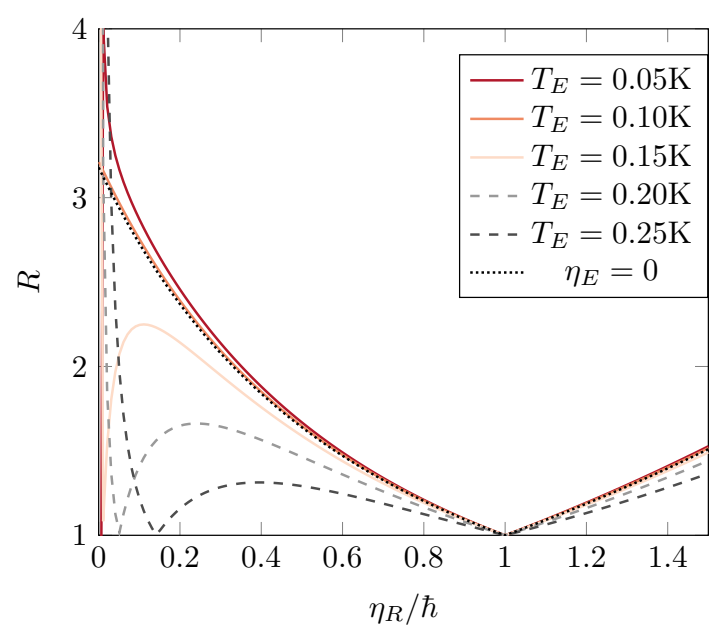

FIG. 5. Influence of a third bath on the rectification index $R$, as defined in (60). The coupling to the third bath is $\eta_{E}=0.1 \hbar, T_{L}=$ $0.1 \mathrm{~K}$, and $T_{R}=0.2 \mathrm{~K}$. The other parameters are given in the caption of Fig. 3.

\section{DISCUSSION}

In this paper we have studied the heat current through a qubit between two thermal baths. Earlier studies performed calculations that were done using the polaron transform [23,26-31], or when explicitly performing the noninteracting blip approximation (NIBA) were focused on the first moment [34]. Here we rederived the explicit expression for the generating function of the heat current by directly performing the NIBA. The Laplace transform of the cumulant generating function of the heat current is a large deviation function (or rate function) that allows one to quantify rare events. In equilibrium, it can be shown that rate functions are simply related to the traditional thermodynamic potentials such as entropy or free energy [71]. Far from equilibrium, large deviation functions can be defined for a large class of dynamical processes and are good candidates for playing the role of generalized potentials [7].

In classical physics, a few exact solutions for the large deviations in some integrable interacting particles models have been found and a nonlinear hydrodynamic theory, known as macroscopic fluctuation theory, has been developed $[7,8]$. In the quantum case, the role of large deviation functions is played by the full counting statistics (FCS) [72-77] for which a path integral formulation akin to macroscopic fluctuation theory has been formulated [78]. The FCS exhibits universal features and phase transitions [79] and obeys the fluctuation theorem [80-83]. However, in the quantum realm, exact results for interacting systems are very rare, among the most noticeable is a series of remarkable calculations performed for the XXZ open spin chain interacting with boundary reservoirs within the Lindblad framework $[84,85]$.

In the present work, our aim was to study the heat transport in the spin-boson model, starting from the microscopic model that embodies the qubit and the reservoirs. We hence do not rely on a Markovian assumption, but eventually that the tunneling element is small, as is inherent to the NIBA.

Our analysis begins with the exact expression of the generating function in terms of a Feynman-Vernon-type path integral from which we derived a full analytical formula for the generating function of the heat current. We recover the earlier results derived using the polaron transform [26,27].

As a numerical example we studied the first moment of the generating function, the heat current. We saw that this shows rectification when the coupling strength of the qubit to both baths is not equal, as was already found by Refs. [23,67]. When the temperature gradient is flipped, the current changes direction, but it does not have the same magnitude in both directions and therefore breaks the Fourier law of heat conduction.

A very important property satisfied by the generating function is the Gallavotti-Cohen fluctuation theorem that embodies at the macroscopic scale the time-reversal invariance of the microscopic dynamics. The fluctuation theorem implies in particular the fluctuation-dissipation relation and the Onsager reciprocity rules when different currents are present $[53,54,57,86,87]$.

The fact that the formal definition of generating function does obey the Gallavotti-Cohen symmetry is rather straightforward to obtain. This relation remains true after 
the NIBA [26,27]. This means that NIBA respects the fundamental symmetries of the underlying model, or equivalently, that the spin-boson problem with NIBA is by itself a thermodynamically consistent model. One consequence is that the fluctuation-dissipation relation is retrieved under the NIBA. Indeed, we explicitly calculated the first and second moment of the heat. When the temperature difference between the baths is small, we found the heat conductance $\kappa$ as the first moment of heat per unit time divided by temperature difference. The variance of the heat at equilibrium, when both temperatures are the same, is then per unit time proportional to $\kappa$. We emphasize that the Gallavotti-Cohen relation is valid far from equilibrium and it implies relations between response coefficients at arbitrary orders [88].

\section{ACKNOWLEDGMENTS}

We gratefully acknowledge discussions with Jukka Pekola, Dmitry Golubev, and Rafael Sanchez. We also thank Hugo Touchette for many useful suggestions. We thank the nordita program "New Directions in Quantum Information" (Stockholm). We thank Nordita, Quantum Technology Finland (Espoo, Finland), and International Centre for Theory of Quantum Technologies (Gdańsk, Poland) for their financial support for this event. The work of E.A. was partially supported by Foundation for Polish Science through Near-term quantum computers: project (Contract No. POIR.04.04.00-00$17 \mathrm{C} 1 / 18-00)$. The work of B.D. is supported by DOMAST.

\section{APPENDIX A: DERIVATION OF EQ. (11)}

The expression for the generating function (9) can be rewritten by defining

$$
\begin{aligned}
\bar{H} & =e^{i\left(\alpha_{R} H_{R}+\alpha_{L} H_{L}\right) / \hbar} H e^{-i\left(\alpha_{R} H_{R}+\alpha_{L} H_{L}\right) / \hbar} \\
& =H_{S}+H_{L}+H_{R}+\bar{H}_{S L}+\bar{H}_{S R}
\end{aligned}
$$

with

$$
\bar{H}_{S L / R}=-\sigma_{z} \sum_{b \in L / R} L_{b} \sqrt{\frac{\hbar}{2 m \omega_{b, L / R}}}\left(b_{b} e^{-i \omega_{b} \alpha_{L / R}}+b_{b}^{\dagger} e^{i \omega_{b} \alpha_{L / R}}\right) .
$$

Let $\bar{U}_{t, \alpha_{R}, \alpha_{L}}$ be the corresponding evolution operator, the generating function (9) is

$$
G_{i, f}(\vec{\alpha}, t)=\operatorname{tr}\left\langle f\left|\bar{U}_{t, \alpha_{R}, \alpha_{L}}\left(\rho_{\beta_{L}} \otimes \rho_{\beta_{R}} \otimes|i\rangle\langle i|\right) U_{t}^{\dagger}\right| f\right\rangle .
$$

With this expression the influence functional can be derived in the usual way [2], leading to (13).

\section{APPENDIX B: SURVIVING TERMS OF THE NIBA}

It is convenient to define the sojourn index

$$
\chi_{t}=X_{t}+Y_{t}
$$

such that during a sojourn $X_{t}=Y_{t}=\frac{1}{2} \chi_{t}$ and the blip index

$$
\xi_{t}=X_{t}-Y_{t} \text {. }
$$

such that during a blip $X_{t}=-Y_{t}=\frac{1}{2} \xi_{t}$. Since we will be performing two time integrals, we will be needing the second primitive functions $K_{i}^{R / L}, K_{r}^{R / L}$ of $k_{i}^{R / L}(t-s)$ and $k_{r}^{R / L}(t-s)$. The second primitive is defined as

$$
K_{i / r}^{R / L}=\int d t d s k_{i / r}^{R / L}(t-s)
$$

Note that the primitive functions have an extra minus sign, due to the fact that we are integrating over $-s$

$$
\begin{aligned}
& K_{i}^{R / L}(t)=\sum_{b} \frac{\left(C_{b, R / L}\right)^{2}}{2 m_{b} \omega_{b, R / L}^{3}} \sin \left(\omega_{b, R / L} t\right) \\
& K_{r}^{R / L}(t)=\sum_{b} \frac{\left(C_{b, R / L}\right)^{2}}{2 m_{b} \omega_{b, R / L}^{3}} \operatorname{coth}\left(\frac{\hbar \omega_{b, R / L} \beta_{R / L}}{2}\right) \cos \left(\omega_{b, R / L} t\right) .
\end{aligned}
$$

\section{Blip-blip}

We consider a blip interval that runs from a time $t^{*}$ to $t^{*}+\Delta t_{b}$.

\section{a. Imaginary part of the action}

Notice that in the same blip interval $X_{t}=X_{s}=-Y_{t}=-Y_{s}$, hence $X_{t} X_{s}=Y_{t} Y_{s}=1$ and $X_{t} Y_{s}=Y_{t} X_{s}=-1$. This means that the term proportional to $X_{t} X_{s}-Y_{t} Y_{s}$ in the imaginary part of the action (15) will not contribute. The remaining terms, which we denote by $R(\vec{\alpha}, t)=R^{R}\left(\alpha_{R}, t\right)+R^{L}\left(\alpha_{L}, t\right)$, give

$$
\begin{aligned}
R^{j}\left(\alpha_{j}, t\right)-\frac{1}{2} K_{i}^{j}\left(\alpha_{j}\right) & =-\frac{1}{4} \int_{t^{*}}^{t^{*}+\Delta t_{B}} \int_{t^{*}}^{t^{*}+\Delta t_{B}} d t d s k_{i}^{j}\left(t-s+\alpha_{j}\right)=\frac{1}{4}\left[K_{i}^{j}\left(\Delta t_{B}+\alpha_{j}\right)+K_{i}^{j}\left(-\Delta t_{B}+\alpha_{j}\right)-2 K_{i}^{j}\left(\alpha_{j}\right)\right] \\
& =\frac{1}{2} \sum_{b} \frac{C_{b, j}^{2}}{2 m_{b, j} \omega_{b, j}^{3}} \sin \left(\omega_{b} \alpha_{j}\right) \cos \left(\omega_{b, j} \Delta t_{B}\right)-\frac{1}{2} K_{i}^{j}\left(\alpha_{j}\right),
\end{aligned}
$$

where $j=R$ or $L$. We isolated the $\frac{1}{2} K_{i}^{j}\left(\alpha_{j}\right)$ term to anticipate a cancellation with sojourn-sojourn terms. 


\section{b. Real part of the action}

For the real part, all terms contribute. The result is $C\left(\vec{\alpha}, \Delta t_{B}\right)=C^{R}\left(\alpha_{R}, \Delta t_{B}\right)+C^{L}\left(\alpha_{L}, \Delta t_{B}\right)$, with

$$
\begin{aligned}
& C^{j}\left(\alpha, \Delta t_{B}\right) \equiv \frac{1}{4} \int_{t^{*}}^{t^{*}+\Delta t_{B}} \int_{t^{*}}^{t} d t d s\left[2 k_{r}^{j}(t-s)+k_{r}(t-s+\alpha)+k_{r}(t-s-\alpha)\right] \\
& =\frac{1}{4}\left[-2 K_{r}^{j}\left(\Delta t_{B}\right)+2 K_{r}^{j}(0)-K_{r}^{j}\left(\Delta t_{B}+\alpha\right)-K_{r}^{j}\left(\Delta t_{B}-\alpha\right)+2 K^{j}(\alpha)\right] \\
& =\frac{1}{2} \sum_{b} \frac{C_{b, j}^{2}}{2 m_{b, j} \omega_{b, j}^{3}} \operatorname{coth}\left(\frac{\omega_{b, j} \hbar \beta}{2}\right)\left[\cos \left(\omega_{b, j} \alpha\right)+1\right]\left[1-\cos \left(\omega_{b, j} \Delta_{B}\right)\right] .
\end{aligned}
$$

\section{Blip-sojourn}

We consider a blip interval running from $t^{*}-\Delta t_{b}$ to $t^{*}$ and the ensuing sojourn interval from $t^{*}$ to $t^{*}+\Delta t_{s}$.

\section{a. Imaginary part}

The contribution from the imaginary part of the action is $\chi \xi X_{-}\left(\vec{\alpha}, \Delta t_{B}\right), X_{-}\left(\vec{\alpha}, \Delta t_{B}\right)=X_{-}^{R}\left(\alpha_{R}, \Delta t_{B}\right)+X_{-}^{L}\left(\alpha_{L}, \Delta t_{B}\right)$ with

$$
\begin{aligned}
X_{-}^{j}\left(\alpha, \Delta t_{B}\right)= & \frac{1}{4} \int_{t^{*}}^{t^{*}+\Delta t_{S}} d t \int_{t^{*}-\Delta t_{B}}^{t^{*}} d s\left[2 k_{i}^{j}(t-s)-k_{i}^{j}(t-s+\alpha)-k_{i}^{j}(t-s-\alpha)\right] \\
= & \frac{1}{4}\left(2 K_{i}^{j}\left(\Delta t_{S}\right)-2 K_{i}^{j}\left(\Delta t_{S}+\Delta t_{B}\right)+2 K_{i}^{j}\left(\Delta t_{B}\right)-2 K_{i}^{j}(0)-K_{i}^{j}\left(\Delta t_{S}+\alpha\right)+K_{i}^{j}\left(\Delta t_{S}+\Delta t_{B}+\alpha\right)\right. \\
& \left.-K_{i}^{j}\left(\Delta t_{B}+\alpha\right)+K_{i}^{j}(\alpha)-K_{i}^{j}\left(\Delta t_{S}-\alpha\right)+K_{i}^{j}\left(\Delta t_{S}+\Delta t_{B}-\alpha\right)-K_{i}^{j}\left(\Delta t_{B}-\alpha\right)+K_{i}^{j}(-\alpha)\right) .
\end{aligned}
$$

Following the NIBA, we have $K_{i}\left(\Delta t_{S}\right)=K_{i}\left(\Delta t_{S}+\Delta t_{B}\right)=K_{i}\left(\Delta t_{S}+\Delta t_{B}+\alpha\right)$, which leads to a significant simplification in the above equation, we find

$$
\begin{aligned}
X_{-}^{j}\left(\alpha, \Delta t_{B}\right) & =\frac{1}{4}\left[2 K_{i}^{j}\left(\Delta t_{B}\right)-K_{i}^{j}\left(\Delta t_{B}-\alpha\right)-K_{i}\left(\Delta t_{B}+\alpha\right)\right] \\
& =\frac{1}{2} \sum \frac{C_{b, j}^{2}}{2 m_{b, j} \omega_{b}^{3}} \sin \left(\omega_{b, j} \Delta t_{B}\right)\left[1-\cos \left(\omega_{b, j} \alpha\right)\right] .
\end{aligned}
$$

\section{b. Real part}

The real part gives $\chi \xi F_{-}\left(\vec{\alpha}, \Delta t_{B}\right), F_{-}\left(\vec{\alpha}, \Delta t_{B}\right)=F_{-}^{R}\left(\alpha_{R}, \Delta t_{B}\right)+F_{-}^{L}\left(\alpha_{L}, \Delta t_{B}\right)$

$$
\begin{aligned}
F_{-}^{j}\left(\alpha, \Delta t_{B}\right)= & \frac{1}{4} \chi \xi \int_{t^{*}}^{t^{*}+\Delta t_{S}} d t \int_{t^{*}-\Delta t_{B}}^{t^{*}} d s\left[k_{r}^{j}(t-s+\alpha)-k_{r}^{j}(t-s-\alpha)\right] \\
= & \frac{1}{4} \chi \xi\left(K_{r}^{j}\left(\Delta t_{S}+\alpha\right)+K_{r}^{j}\left(\Delta t_{B}+\alpha\right)-K_{r}^{j}\left(\Delta t_{B}+\Delta t_{S}+\alpha\right)-K_{r}^{j}(\alpha)\right. \\
& \left.-K_{r}^{j}\left(\Delta t_{S}-\alpha\right)-K_{r}^{j}\left(\Delta t_{B}-\alpha\right)+K_{r}^{j}\left(\Delta t_{B}+\Delta t_{S}-\alpha\right)+K_{r}^{j}(-\alpha)\right) .
\end{aligned}
$$

Under the same argument as for the imaginary part, we get

$$
\begin{aligned}
F_{-}^{j}\left(\alpha, \Delta t_{B}\right) & =\frac{1}{4}\left(K_{r}^{j}\left(\Delta t_{B}+\alpha\right)-K_{r}^{j}\left(\Delta t_{B}-\alpha\right)\right) \\
& =-\frac{1}{2} \sum_{b} \frac{\left(C_{b, j}\right)^{2}}{2 m_{b} \omega_{b}^{3}} \operatorname{coth}\left(\frac{\omega_{b} \hbar \beta}{2}\right) \sin \left(\omega_{b} \Delta t_{B}\right) \sin \left(\omega_{b} \alpha\right) .
\end{aligned}
$$

\section{Sojourn-blip}

The blip interval runs from $t^{*}-\Delta t_{s}$ to $t^{*}$ and the blip interval $t^{*}$ to $t^{*}+\Delta t_{b}$. 


\section{a. Imaginary part}

This calculation is similar to the blip-sojourn term, but with less cancelations.

$$
\begin{aligned}
X_{+}^{j}\left(\alpha, \Delta t_{b}\right)= & \frac{1}{4} \int_{t^{*}}^{t^{*}+\Delta t_{B}} d t \int_{t^{*}-\Delta t_{S}}^{t^{*}} d s\left[2 k_{i}^{j}(t-s)+k_{i}^{j}(t-s+\alpha)+k_{i}^{j}(t-s-\alpha)\right] \\
= & \frac{1}{4}\left(2 K_{i}^{j}\left(\Delta t_{S}\right)-2 K_{i}^{j}\left(\Delta t_{S}+\Delta t_{B}\right)+2 K_{i}^{j}\left(\Delta t_{B}\right)-2 K_{i}^{j}(0)+K_{i}^{j}\left(\Delta t_{S}+\alpha\right)-K_{i}^{j}\left(\Delta t_{S}+\Delta t_{B}+\alpha\right)\right. \\
& \left.+K_{i}^{j}\left(\Delta t_{B}+\alpha\right)-K_{i}^{j}(\alpha)+K_{i}^{j}\left(\Delta t_{S}-\alpha\right)-K_{i}^{j}\left(\Delta t_{S}+\Delta t_{B}-\alpha\right)+K_{i}^{j}\left(\Delta t_{B}-\alpha\right)-K_{i}^{j}(-\alpha)\right) .
\end{aligned}
$$

Again, under NIBA, we have $K_{i}^{j}\left(\Delta t_{S}\right)=K_{i}^{j}\left(\Delta t_{S}\right)=K_{i}^{j}\left(\Delta t_{S}+\Delta t_{B}\right) K_{i}^{j}\left(\Delta t_{S}+\Delta t_{B}+\alpha\right)$, which gives

$$
X^{+}\left(\alpha, \Delta t_{b}\right)=\frac{1}{4}\left[2 K_{i}^{j}\left(\Delta t_{B}\right)+K_{i}^{j}\left(\Delta t_{B}-\alpha\right)+K_{i}^{j}\left(\Delta t_{B}+\alpha\right)\right]=\frac{1}{2} \sum \frac{\left(C_{b, j}\right)^{2}}{2 m_{b} \omega_{b}^{3}} \sin \left(\omega_{b} \Delta t_{B}\right)\left[1+\cos \left(\omega_{b} \alpha\right)\right] .
$$

\section{b. Real part}

$$
\begin{aligned}
F_{+}^{j}\left(\alpha, \Delta t_{b}\right)= & \frac{1}{4} \chi \xi \int_{t^{*}}^{t^{*}+\Delta t_{S}} d t \int_{t^{*}-\Delta t_{B}}^{t^{*}} d s\left[-k_{r}^{j}(t-s+\alpha)+k_{r}^{j}(t-s-\alpha)\right] \\
= & \chi \xi \frac{1}{4}\left(-K_{r}^{j}\left(\Delta t_{S}+\alpha\right)-K_{r}^{j}\left(\Delta t_{B}+\alpha\right)+K_{r}^{j}\left(\Delta t_{B}+\Delta t_{S}+\alpha\right)+K_{r}^{j}(\alpha)\right. \\
& \left.+K_{r}^{j}\left(\Delta t_{S}-\alpha\right)+K_{r}^{j}\left(\Delta t_{B}-\alpha\right)-K_{r}^{j}\left(\Delta t_{B}+\Delta t_{S}-\alpha\right)-K_{r}^{j}(-\alpha)\right) .
\end{aligned}
$$

Under the same argument as for the imaginary part, we get

$$
F_{+}^{j}\left(\alpha, \Delta t_{b}\right)=\frac{1}{4}\left(-K_{r}^{j}\left(\Delta t_{B}+\alpha\right)+K_{r}^{j}\left(\Delta t_{B}-\alpha\right)\right)=\frac{1}{2} \sum \frac{\left(C_{b, j}\right)^{2}}{2 m_{b} \omega_{b}^{3}} \operatorname{coth}\left(\frac{\omega_{b} \hbar \beta}{2}\right) \sin \left(\omega_{b} \Delta t_{B}\right) \sin \left(\omega_{b} \alpha\right) .
$$

Note that $F_{+}=-F_{-}$.

\section{Sojourn-sojourn}

The first sojourn interval runs from $t^{*}$ to $t^{*}+\Delta t_{S_{1}}$ and the blip interval $t^{*}+\Delta t_{S_{1}}$ to $t^{*}+\Delta t_{S_{1}}+\Delta t_{S_{2}}$.

\section{a. Imaginary part}

We find

$$
\begin{aligned}
B^{j}(\alpha) & \equiv \frac{1}{4} \int_{t^{*}}^{t^{*}+\Delta t_{s}} \int_{t^{*}}^{t^{*}+\Delta t_{s}} d t d s k_{i}^{j}(t-s+\alpha)=\frac{1}{4}\left(2 K_{i}^{j}(\alpha)-K_{i}^{j}\left(\Delta t_{s}+\alpha\right)-K_{i}^{j}\left(-\Delta t_{s}+\alpha\right)\right) \\
& =\frac{1}{2} K_{i}^{j}(\alpha)=\frac{1}{2} \sum_{b} \frac{\left(C_{b, j}\right)^{2}}{2 m_{b} \omega_{b}^{3}} \sin (\alpha) .
\end{aligned}
$$

\section{b. Real part}

$$
\begin{aligned}
D^{j}(\alpha) & =\frac{1}{4} \int_{t^{*}}^{t^{*}+\Delta t_{S}} d t \int_{t^{*}}^{t} d s\left(2 k_{r}^{j}(t-s)-k_{r}^{j}(t-s+\alpha)-k_{r}^{j}(t-s-\alpha)\right) \\
& =\frac{1}{4}\left(2 K_{r}^{j}(0)-2 K_{r}^{j}\left(\Delta t_{S}\right)+K_{r}^{j}\left(\Delta t_{S}+\hbar \alpha\right)-K_{r}^{j}(\alpha)+K_{r}^{j}\left(\Delta t_{S}-\hbar \alpha\right)-K_{r}^{j}(-\alpha)\right)=\frac{1}{2}\left[K_{r}^{j}(0)-K_{r}^{j}(\alpha)\right] \\
& =\frac{1}{2} \sum \frac{\left(C_{b, j}\right)^{2}}{2 m_{b} \omega_{b}^{3}} \operatorname{coth}\left(\frac{\omega_{b} \hbar \beta_{j}}{2}\right)[1-\cos (\alpha)] .
\end{aligned}
$$

There will also be cancellations between $D$ and $C$.

\section{Sojourn-(blip)-sojourn}

The first sojourn interval runs from $t^{*}$ to $t^{*}+\Delta t_{S_{1}}$ and the second sojourn interval from $t^{*}+\Delta t_{S_{1}}+\Delta t_{B}$ to $t^{*}+\Delta t_{S_{1}}+$ $\Delta t_{B_{1}}+\Delta t_{S_{2}}$, where $\Delta t_{B}$ is the duration of the blip. 


\section{a. Imaginary part}

$$
\begin{aligned}
\Lambda^{j}\left(\alpha, \Delta t_{B}\right) & =\frac{1}{4} \int_{t^{*}+\Delta t_{S_{1}}+\Delta t_{B}}^{t^{*}+\Delta t_{S_{1}}+\Delta t_{B}+\Delta t_{S_{2}}} d t \int_{t^{*}}^{t^{*}+\Delta t_{S_{1}}} d s\left[k_{i}^{j}(t-s+\alpha)-k_{i}^{j}(t-s-\alpha)\right] \\
& =\frac{1}{4}\left[-K_{i}^{j}\left(\Delta t_{B}+\alpha\right)+K_{i}^{j}\left(\Delta t_{B}-\alpha\right)\right]=-\frac{1}{2} \sum_{b} \frac{\left(C_{b, j}\right)^{2}}{2 m_{b} \omega_{b}^{3}} \cos \left(\omega_{b} \Delta t_{B}\right) \sin \left(\omega_{b} \alpha\right) .
\end{aligned}
$$

\section{b. Real part}

$$
\begin{aligned}
\Sigma^{j}\left(\alpha, \Delta t_{B}\right) & =\frac{1}{4} \int_{t^{*}+\Delta t_{S_{1}}+\Delta t_{B}}^{t^{*}+\Delta t_{S_{1}}+\Delta t_{B}+\Delta t_{S_{2}}} d t \int_{t^{*}}^{t^{*}+\Delta t_{S_{1}}} d s\left[2 k_{r}^{j}(t-s)-k_{r}^{j}(t-s+\alpha)-k_{r}^{j}(t-s-\alpha)\right] \\
& =\frac{1}{4}\left[K_{r}^{j}\left(\Delta t_{B}+\alpha\right)+K_{r}^{j}\left(\Delta t_{B}-\alpha\right)-2 K_{r}^{j}\left(\Delta t_{B}\right)\right] \\
& =\frac{1}{2} \sum \frac{\left(C_{b, j}\right)^{2}}{2 m_{b, j} \omega_{b, j}^{3}} \operatorname{coth}\left(\frac{\omega_{b, j} \hbar \beta_{j}}{2}\right) \cos \left(\omega_{b, j} \Delta t_{B}\right)\left[\cos \left(\omega_{b, j} \alpha\right)-1\right] .
\end{aligned}
$$

\section{Transfer matrix}

The generating function, using the terms calculated in the previous sections, is

$$
\begin{aligned}
G_{S \rightarrow S}(\alpha)= & \sum_{n=0}^{+\infty}(-1)^{n}\left(\frac{\Delta}{2}\right)^{n} \int d t_{1} \ldots d t_{2 n} \sum_{\substack{\chi_{1}, \ldots, \chi_{n}= \pm 1, \xi_{1}, \ldots, \xi_{n}= \pm 1}} \exp \left(-\frac{i}{\hbar} \epsilon \sum_{i} \xi_{i}\left(t_{2 i}-t_{2 i-1}\right)\right) \\
& \times \exp \left(\frac{i}{\hbar} \sum_{j=R, L} \sum_{i} \chi_{i} \xi_{i+1} X_{+}^{j}\left(\alpha_{j}, \Delta_{2 i+2}\right)+\chi_{i} \xi_{i} X_{-}^{j}\left(\alpha_{j}, \Delta_{2 i}\right)+\chi_{i} \chi_{i+1} \Lambda^{j}\left(\alpha, \Delta_{2 i+2}\right)+R^{j}\left(\alpha, \Delta_{2 i}\right)\right) \\
& \times \exp \left(-\frac{1}{\hbar} \sum_{j=R, L} \sum_{i} \chi_{j} \xi_{i+1} F_{+}^{j}\left(\alpha_{j}, \Delta_{2 i+2}\right)+\chi_{i} \xi_{i} F_{-}^{j}\left(\alpha_{j}, \Delta_{2 i}\right)+\chi_{i} \chi_{i+1} \Sigma^{j}\left(\alpha_{j}, \Delta_{2 i+2}\right)+C^{\prime}\left(\alpha_{j}, \Delta_{2 i}\right)\right) .
\end{aligned}
$$

To express the resulting generating function in terms of a transfer matrix, it is convenient to first define for $j=R$ or $L$

$$
\begin{gathered}
Z_{j}^{+}(t)=X_{+}^{j}(\alpha, t)+X_{-}^{j}(\alpha, t)=\frac{2 \eta_{j}}{\pi} \int_{0}^{\Omega} d \omega \frac{1}{\omega} \sin (\omega t) \\
Z_{j}^{-}(\alpha, t)=X_{+}^{j}(\alpha, t)-X_{-}^{j}(\alpha, t)=\frac{2 \eta_{j}}{\pi} \int_{0}^{\Omega} d \omega \frac{1}{\omega} \sin (\omega t) \cos (\omega \alpha)
\end{gathered}
$$

and

$$
\begin{gathered}
\Gamma_{j}^{+}(t)=C^{j}(\alpha, t)+D^{j}(\alpha, t)+\Sigma(\alpha, t)=\frac{2 \eta_{j}}{\pi} \int_{0}^{\Omega} d \omega \frac{1}{\omega} \operatorname{coth}\left(\frac{\omega \hbar \beta_{j}}{2}\right)[1-\cos (\omega t)] \\
\Gamma_{j}^{-}(\alpha, t)=C^{j}(\alpha, t)+D^{j}(\alpha, t)-\Sigma(\alpha, t)=\frac{2 \eta_{j}}{\pi} \int_{0}^{\Omega} d \omega \frac{1}{\omega} \operatorname{coth}\left(\frac{\omega \hbar \beta_{j}}{2}\right)[1-\cos (\omega t) \cos (\omega \alpha)],
\end{gathered}
$$

which allows us to write the generating function as (18).

\section{APPENDIX C: HEAT CURRENT}

In this section we are interested in studying the heat current between the two baths. The heat current is defined as

$$
\Pi\left(\beta_{C}, \beta_{R}\right)=\lim _{t \rightarrow \infty} \frac{\left\langle\Delta E_{c}\right\rangle}{t},
$$

where $\beta_{L}$ and $\beta_{R}$ are the inverse temperatures of respectively the left bath and the right bath, and $E_{c}$ is the energy of the left bath.

To our knowledge the results in this section were first obtained in Ref. [89] although only stated for the case of zero level splitting. Here we rederive them using the same notation as in the main body of the paper. We will show that 
$\Pi(\beta, \beta)=0$, which one would physically expect. It means that in the steady state there is no heat transfer between two baths with the same temperature. Furthermore, we calculate the thermal conductance $\kappa$, which is defined by the expansion for small temperature differences $\Delta \beta$ in both baths

$$
\Pi(\beta, \beta+\Delta \beta)=\kappa \Delta \beta+O\left(\Delta \beta^{2}\right) .
$$

Our starting point is a result by the authors of Ref. [34] for the form of the heat current

$$
\Pi=\left(\frac{\Delta}{2}\right)^{2}\left(\frac{p_{-}}{p_{+}+p_{-}} \pi_{\uparrow}+\frac{p_{+}}{p_{+}+p_{-}} \pi_{\downarrow}\right),
$$

where $\frac{p_{-}}{p_{+}+p_{-}}$is the steady-state population of the lower qubit state and $\left(\frac{\Delta}{2}\right)^{2} \pi_{\uparrow}$ the heat current related to this state.

Let us introduce the characteristic functions

$$
\begin{aligned}
& C_{L}(t)=e^{-\frac{1}{\hbar} \Gamma_{L}^{+}(t)+\frac{i}{\hbar} Z_{L}^{+}(t)} \\
& C_{R}(t)=e^{-\frac{1}{\hbar} \Gamma_{R}^{+}(t)+\frac{i}{\hbar} Z_{R}^{+}(t)},
\end{aligned}
$$

which allow us to conveniently write the coefficients of (C3)

$$
\begin{gathered}
p_{+}=\int_{-\infty}^{\infty} d t C_{L}(t) C_{R}(t) e^{i \epsilon t} \\
p_{-}=\int_{-\infty}^{\infty} d t C_{L}(t) C_{R}(t) e^{-i \epsilon t}, \\
\pi_{\uparrow}=-i \hbar \int_{-\infty}^{\infty} d t \frac{d C_{L}(t)}{d t} C_{R}(t) e^{i \epsilon t} \\
\pi_{\downarrow}=-i \hbar \int_{-\infty}^{\infty} d t \frac{d C_{L}^{+}(t)}{d t} C_{R}^{+}(t) e^{-i \epsilon t},
\end{gathered}
$$

and

$$
\begin{aligned}
& \Sigma^{+}=-\hbar^{2} \int_{-\infty}^{\infty} d t \frac{d^{2} C_{L}(t)}{d t^{2}} C_{R}(t) e^{i \epsilon t} \\
& \Sigma^{-}=-\hbar^{2} \int_{-\infty}^{\infty} d t \frac{d^{2} C_{L}(t)}{d t^{2}} C_{R}(t) e^{-i \epsilon t} .
\end{aligned}
$$

\section{Two baths with the same temperatures}

When both baths have the same temperatures, we expect the steady-state heat transfer to be zero

$$
\Pi(\beta, \beta)=0 \text {. }
$$

Via an analytic continuation argument outlined in Appendix D, we find that

$$
p_{+}\left(\beta_{L}, \beta_{R}\right)=\frac{1}{2} \int d t C_{L}(t+i \Delta \beta \hbar) C_{R}(t) e^{-\frac{i}{\hbar} \epsilon t} e^{-\epsilon \beta_{R}}
$$

and

$$
\pi_{\downarrow}\left(\beta_{C}, \beta_{R}\right)=i \hbar \int d t \frac{d C_{L}(t+i \Delta \beta \hbar)}{d t} C_{R}(t) e^{\frac{i}{\hbar} \epsilon t} e^{\epsilon \beta_{R}}
$$

with $\Delta \beta=\beta_{C}-\beta_{R}$. When both temperatures are equal, these relations transform to

$$
p_{+}(\beta, \beta)=e^{-\epsilon \beta} p_{-}(\beta, \beta)
$$

and

$$
\pi_{\uparrow}(\beta, \beta)=-e^{-\epsilon \beta} \pi_{\downarrow}(\beta, \beta) .
$$

Equations (C12) and (C13) directly give us

$$
\begin{aligned}
\Pi(\beta, \beta) & =\left(\frac{\Delta}{2}\right)^{2} \frac{1}{p_{+}+p_{-}}\left(p_{-} \pi_{\uparrow}+p_{+} \pi_{\downarrow}\right) \\
& =\left(\frac{\Delta}{2}\right)^{2} \frac{e^{-\epsilon \beta}}{p_{+}+p_{-}}\left(-p_{-} \pi_{\downarrow}+p_{-} \pi_{\downarrow}\right)=0 .
\end{aligned}
$$

\section{Thermal conductance}

The obtain an explicit formula for the thermal conductance $\kappa$ one should expand (C3) in the difference between the temperature of both baths $\Delta \beta=\beta_{L}-\beta_{R}$. Differentiating the denominator $(A+D)$ gives no contribution as it multiplies a parenthesis $D \pi_{\uparrow}+A \pi_{\downarrow}$, which vanishes to zeroth order. We can therefore write

$$
\begin{gathered}
\kappa=\left(\frac{\Delta}{2}\right)^{2} \frac{1}{p_{+}+p_{-}}\left(\partial_{\beta_{L}}\left(p_{-}\right) \pi_{\uparrow}+p_{-} \partial_{\beta_{L}}\left(\pi_{\uparrow}\right)\right. \\
\left.+\partial_{\beta_{L}}\left(p_{+}\right) \pi_{\downarrow}+p_{+} \partial_{\beta_{L}}\left(\pi_{\downarrow}\right)\right) .
\end{gathered}
$$

All terms on the right-hand side are evaluated at $\beta_{L}=\beta_{R}=\beta$.

The calculation of $\kappa$ is presented in Appendix E. The idea of the calculation is to write out $\partial_{\beta}\left(p_{-}\right) \pi_{\uparrow}$ and $p_{-} \partial_{\beta}\left(\pi_{\uparrow}\right)$, and to keep track how the terms generated in the partial derivatives $\partial_{\beta}\left(p_{-}\right)$and and $\partial_{\beta}\left(\pi_{\uparrow}\right)$ change as the integral variable $t$ is shifted to $t+i \hbar \beta$. The result is

$$
\kappa=\left(\frac{\Delta}{2}\right)^{2} \frac{1}{p_{+}+p_{-}}\left(p_{+} \Sigma^{-}+4 \pi_{\downarrow} \pi_{\uparrow}+p_{-} \Sigma^{+}\right),
$$

where $\tilde{C}$ and $\tilde{D}$ are the Laplace transforms of the matrix elements defined in Eq. (20) and the accent denotes the derivative to the first variable.

\section{APPENDIX D: ANALYTIC CONTINUATION}

Suppose that all functions are analytical in the strip $0 \leqslant$ $\Im t \leqslant \hbar \beta_{R}$ [note that in Appendix E of Ref. [62] the authors assume an analytic continuation to negative imaginary values of $t$; however, they consider the function $G(t)$ related to the function $C(t)$ by $G(t)=e^{-C(t)}$ ]. Then any of the integrals, say $D$, can be written as

$$
p_{+}=\int d t C_{L}\left(t+i \beta_{R} \hbar\right) C_{R}\left(t+i \beta_{R} \hbar\right) e^{\frac{i}{\hbar} \epsilon\left(t+i \beta_{R} \hbar\right)} .
$$

The exponents in $C_{L}$ and $C_{R}$ are sums over bath oscillators. Each oscillator $b$ contributes

$$
\text { Term }=\frac{1}{2 m_{b} \omega_{b}}\left(-\operatorname{coth} \frac{\omega_{b} \hbar \beta}{2}\left(1-\cos \omega_{b} t\right)+i \cdot \sin \omega_{b} t\right),
$$

where $\beta$ is $\beta_{R}$ or $\beta_{L}$. Evaluating first oscillators in the right bath gives

$$
\cos \omega_{b}(t+i \hbar \beta)=\cos \omega_{b} t \cosh \hbar \omega_{b} \beta-i \sin \omega_{b} t \sinh \hbar \omega_{b} \beta
$$

$\sin \omega_{b}(t+i \hbar \beta)=\sin \omega_{b} t \cosh \hbar \omega_{b} \beta+i \cos \omega_{b} t \sinh \hbar \omega_{b} \beta$, 
which with coth $\frac{\omega_{b} \hbar \beta_{R}}{2}$ from above can be combined into

$$
\begin{aligned}
& \cos \omega_{b} t\left(\operatorname{coth} \frac{\omega_{b} \hbar \beta_{R}}{2} \cosh \hbar \omega_{b} \beta_{R}-\sinh \hbar \omega_{b} \beta_{R}\right) \\
& =\cos \omega_{b} t \operatorname{coth} \frac{\omega_{b} \hbar \beta_{R}}{2} \\
& i \sin \omega_{b} t\left(-\operatorname{coth} \frac{\omega_{b} \hbar \beta_{R}}{2} \sinh \hbar \omega_{b} \beta_{R}+\cosh \hbar \omega_{b} \beta_{R}\right) \\
& =-i \sin \omega_{b} t .
\end{aligned}
$$

Hence

$$
C_{R}\left(t+i \beta_{R} \hbar\right)=\overline{C_{R}(t)}=C_{R}(-t)
$$

For the oscillators in the left bath we consider $\left(\Delta \beta=\beta_{L}-\beta_{R}\right)$

$$
\begin{aligned}
C_{L}\left(t+i \beta_{R} \hbar\right) & =C_{L}\left(t-i \hbar \Delta \beta+i \beta_{L} \hbar\right)=\overline{L_{C}(t-i \hbar \Delta \beta)} \\
& =C_{L}(-t+i \hbar \Delta \beta) .
\end{aligned}
$$

Inserting back into the expression for $D$ this means

$$
p_{+}\left(\beta_{L}, \beta_{R}\right)=\int d t C_{L}(-t+i \Delta \beta \hbar) C_{R}(-t) e^{\frac{i}{\hbar} \epsilon t} e^{-\epsilon \beta_{R}} .
$$

\section{APPENDIX E: THERMAL CONDUCTANCE}

\section{Partial derivative of $D$}

For $p_{+}$one finds

$$
\partial_{\beta_{L}} p_{+}=\int d t \partial_{\beta_{L}}\left[\log C_{L}(t)\right]_{\beta_{L}=\beta} C_{L}(t) C_{R}(t) e^{\frac{i}{\hbar} \epsilon t},
$$

where

$$
\partial_{\beta_{C}} \log C_{L}(t)=\sum_{b \in C} \frac{1}{2 m_{b} \omega_{b}}\left(1-\cos \omega_{b} t\right) \frac{1}{\sinh ^{2} \frac{\omega_{b} \hbar \beta_{C}}{2}} \frac{\omega_{b} \hbar}{2} .
$$

Changing $t$ to $t+i \hbar \beta$ will change $C_{L}(t) C_{R}(t) e^{\frac{i}{\hbar} \epsilon t}$ to $C_{L}(-t) C_{R}(-t) e^{-\frac{i}{\hbar} \epsilon(-t)} e^{-\epsilon \beta}$, similarly as in Appendix D.

The logarithmic derivative on the other hand changes in the convenient way:

$$
\begin{aligned}
\partial_{\beta_{L}} \log C_{L}\left(t+i \hbar \beta ; \beta_{L}=\beta\right)= & \sum_{b \in L} \frac{\omega_{b} \hbar}{4 m_{b} \omega_{b}}\left(1-\cos \omega_{b} t\right) \frac{1}{\sinh ^{2} \frac{\omega_{b} \hbar \beta}{2}}+\sum_{b \in L} \frac{\omega_{b} \hbar}{4 m_{b} \omega_{b}} \cos \omega_{b} t(-2) \\
& +\sum_{b \in L} \frac{\omega_{b} \hbar}{4 m_{b} \omega_{b}} \sin \omega_{b} t(2 i) \operatorname{coth} \frac{\omega_{b} \hbar \beta}{2} .
\end{aligned}
$$

The two last terms can be compared to

$$
\begin{aligned}
\partial_{t} \log C_{L}(t) & =\partial_{t}\left(\sum_{b \in L} \frac{1}{2 m_{b} \omega_{b}}\left[-\left(1-\cos \omega_{b} t\right) \operatorname{coth} \frac{\omega_{b} \hbar \beta}{2}+i \sin \omega_{b} t\right]\right) \\
& =\sum_{b \in L} \frac{1}{2 m_{b} \omega_{b}}\left[\left(-\omega_{b} \sin \omega_{b} t\right) \omega_{b} \operatorname{coth} \frac{\omega_{b} \hbar \beta}{2}+i \omega_{b} \cos \omega_{b} t\right] .
\end{aligned}
$$

Equation (E2) can therefore be rewritten as

$$
\partial_{\beta_{L}} \log C_{L}\left(t+i \hbar \beta ; \beta_{L}=\beta\right)=\partial_{\beta_{L}} \log C_{L}\left(-t ; \beta_{L}=\beta\right)+\left.i \hbar \partial_{s} \log C_{L}\left(s ; \beta_{L}=\beta\right)\right|_{s=-t} .
$$

We can now change the integral variable from $t$ to $-t$, which gives

$$
\partial_{\beta}\left(p_{+}\right) \pi_{\downarrow}=-\partial_{\beta}\left(p_{-}\right) \pi_{\uparrow}-\hbar^{2} \int d t \partial_{t}\left(C_{L}(t)\right) C_{R}(t) e^{\frac{i}{\hbar} \epsilon t} \int d t \partial_{t}\left(C_{L}(t)\right) C_{R}(t) e^{-\frac{i}{\hbar} \epsilon t} .
$$

The sign is determined as follows: $\pi_{\uparrow}$ changes sign when it goes to $\pi_{\downarrow}$, but $D$ does not. There is factor $i \hbar$ in the definition of $\pi_{\uparrow}$ and another one in the second term in $\partial_{\beta_{L}} \log C_{L}(t+i \hbar \beta)$. Taken together this gives $-(i \hbar)(-i \hbar)=-\hbar^{2}$.

\section{Partial derivative of $\pi_{\uparrow}$}

This term can be evaluated in practically the same way as the other one. One starts from

$$
\partial_{\beta} \pi_{\downarrow}=-i \partial_{\beta}\left(\int \cdots \partial_{t}\left[C_{L}(t)\right] \cdots\right)=-i\left(\int \cdots C_{L}(t) \partial_{\beta}\left[\log C_{L}(t)\right] \partial_{t}\left[\log C_{L}(t)\right] \cdots\right)-i\left(\int \cdots C_{L}(t) \partial_{t \beta}^{2}\left[\log C_{L}(t)\right] \cdots\right) .
$$

One now treats $\partial_{\beta}\left[\log C_{L}(t)\right]$ in the same way as in (E4). The first term will then give something proportional to $\left\{\partial_{t}\left[\log C_{L}(t)\right]\right\}^{2}$ and the second something proportional to $\partial_{t t}\left[\log C_{L}(t)\right]$. Combining we have

$$
C_{L}\left(\left\{\partial_{t}\left[\log C_{L}(t)\right]\right\}^{2}+\partial_{t t}\left[\log C_{L}(t)\right]\right)=\partial_{t t} C_{L} .
$$

This means that we can write

$$
p_{+} \partial_{\beta}\left(\pi_{\downarrow}\right)=-p_{-} \partial_{\beta}\left(\pi_{\uparrow}\right)-\hbar^{2} \int d t \partial_{t t}\left(C_{L}(t)\right) C_{R}(t) e^{\frac{i}{\hbar} \epsilon t} \int d t C_{L}(t) C_{R}(t) e^{-\frac{i}{\hbar} \epsilon t}
$$


The sign is determined as follows: $\pi_{\downarrow}$ changes sign when it goes to $\pi_{\uparrow}$, but the terms with two time derivates do not change sign. The factors $i \hbar$ and $-i \hbar$ are the same as before.

\section{Combination}

Inserting (E5) and (E8), using that

$$
\begin{aligned}
& \frac{1}{2} \int d t \partial_{t t}\left[C_{L}(t)\right] C_{R}(t) e^{\frac{i}{\hbar} \epsilon t}=\tilde{C}^{\prime \prime}(0,0) \\
& \frac{1}{2} \int d t \partial_{t t}\left[C_{L}(t)\right] C_{R}(t) e^{\frac{-i}{\hbar} \epsilon t}=\tilde{B}^{\prime \prime}(0,0)
\end{aligned}
$$

and symmetrizing one has

$$
\kappa=-\frac{(\hbar \Delta)^{2}}{4\left(p_{+}+p_{-}\right)}\left[p_{+} \tilde{B}^{\prime \prime}(0,0)+4 \tilde{B}^{\prime}(0,0) \tilde{C}^{\prime}(0,0)+p_{-} \tilde{C}^{\prime \prime}(0,0)\right]
$$

[1] A. Kamenev, Field Theory of Non-Equilibrium Systems (Cambridge University Press, Cambridge, 2011).

[2] U. Weiss, Quantum Dissipative Systems (World Scientific, Singapore, 1993).

[3] S. Haroche and J.-M. Raimond, Exploring the Quantum: Atoms, Cavities and Photons (Oxford University Press, Oxford, 2006).

[4] H. P. Breuer and F. Petruccione, The Theory of Open Quantum Systems (Clarendon Press Oxford, 2002).

[5] A. Rivas and S. F. Huelga, Open Quantum System: An Introduction (Springer, Berlin, 2012).

[6] M. T. Mitchison and B. M. Plenio, New J. Phys. 20, 033005 (2018).

[7] B. Derrida, J. Stat. Mech.: Theor. Exp. (2007) P07023.

[8] G. Jona-Lasinio, J. Stat. Mech.: Theor. Exp. (2014) P02004.

[9] J. P. Pekola, P. Solinas, A. Shnirman, and D. V. Averin, New J. Phys. 15, 115006 (2013).

[10] J. P. Pekola, Nature Phys. 11, 118 (2015).

[11] S. Gasparinetti, K. L. Viisanen, O.-P. Saira, T. Faivre, M. Arzeo, M. Meschke, and J. P. Pekola, Phys. Rev. Appl 3, 014007 (2015).

[12] S. Vinjanampathy and J. Anders, Contemp. Phys. 57, 545 (2016).

[13] J. P. Pekola and B. Karimi, J. Low. Temp. Phys. 191, 373 (2018).

[14] B. Karimi and J. Pekola, Phys. Rev. B 95, 019903 (2017).

[15] A. Ronzani, B. Karimi, J. Senior, Y.-C. Chang, J. T. Peltonen, C. Chen, and J. P. Pekola, Nature Phys. 14, 991 (2018).

[16] J. Senior, A. Gubaydullin, B. Karimi, J. Peltonen, J. Ankerhold, and J. Pekola, Commun. Phys. 3, 40 (2020).

[17] S. Gasparinetti, P. Solinas, A. Braggio, and M. Sassetti, New J. Phys. 16, 115001 (2014).

[18] M. Campisi, J. Pekola, and R. Fazio, New J. Phys. 17, 035012 (2015).

[19] B. Donvil, J. Stat. Mech.: Theor. Exp. (2018) 043104.

[20] B. Donvil, P. Muratore-Ginanneschi, J. P. Pekola, and K. Schwieger, Phys. Rev. A 97, 052107 (2018).

[21] G. Lindblad, Commun. Math. Phys. 48, 119 (1976).

[22] J. Tourila, J. Stockburger, T. Ala-Nissila, J. Ankerhold, and M. Möttönen, Phys. Rev. Research 1, 013004 (2019).

[23] D. Segal and A. Nitzan, J. Chem. Phys. 122, 194704 (2005).
[24] C. Aslangul, N. Pottier, and D. Saint-James, J. Phys. 47, 757 (1986).

[25] H. Dekker, Phys. Rev. A 35, 1436 (1987).

[26] L. Nicolin and D. Segal, J. Chem. Phys. 135, 164106 (2011).

[27] L. Nicolin and D. Segal, Phys. Rev. B 84, 161414(R) (2011).

[28] H. M. Friedman, B. K. Agarwalla, and D. Segal, New J. Phys. 20, 083026 (2018).

[29] C. Wang, J. Ren, and J. Cao, Sci. Rep. 5, 11787 (2015).

[30] C. Wang, J. Ren, and J. Cao, Phys. Rev. A 95, 023610 (2017).

[31] J. Liu, C.-Y. Hsieh, C. Wu, and J. Cao, J. Chem. Phys. 148, 234104 (2018).

[32] M. Carrega, P. Solinas, M. Sassetti, and U. Weiss, Phys. Rev. Lett. 116, 240403 (2016).

[33] T. Motz, M. Wiedmann, J. T. Stockburger, and J. Ankerhold, New J. Phys. 20, 113020 (2018).

[34] E. Aurell and F. Montana, Phys. Rev. E 99, 042130 (2019).

[35] Y. Tanimura and R. Kubo, J. Phys. Soc. Jpn. 58, 101 (1989).

[36] Y. Tanimura, J. Chem. Phys. 141, 044114 (2014).

[37] Y. Tanimura, J. Chem. Phys. 142, 144110 (2015).

[38] A. Kato and Y. Tanimura, J. Chem. Phys. 143, 064107 (2015).

[39] A. Kato and Y. Tanimura, J. Chem. Phys. 145, 224105 (2016).

[40] I. de Vega and D. Alonso, Rev. Mod. Phys. 89, 015001 (2017).

[41] N. Makri, J. Phys. Chem. A 102, 4414 (1998).

[42] N. Boudjada and D. Segal, J. Phys. Chem. A 118, 11323 (2014).

[43] M. Kilgour, B. K. Agarwalla, and D. Segal, J. Chem. Phys. 150, 084111 (2019).

[44] K. A. Velizhanin, H. Wang, and M. Thoss, Chem. Phys. Lett. 460, 325 (2008).

[45] J. T. Stockburger and C. H. Mak, J. Chem. Phys. 110, 4983 (1999).

[46] K. Saito and T. Kato, Phys. Rev. Lett. 111, 214301 (2013).

[47] M. Esposito, M. A. Ochoa, and M. Galperin, Phys. Rev. B 92, 235440 (2015).

[48] D. Newman, F. Mintert, and A. Nazir, Phys. Rev. E 95, 032139 (2017).

[49] W. Dou, M. A. Ochoa, A. Nitzan, and J. E. Subotnik, Phys. Rev. B 98, 134306 (2018).

[50] M. Perarnau-Llobet, H. Wilming, A. Riera, R. Gallego, and J. Eisert, Phys. Rev. Lett. 120, 120602 (2018). 
[51] C. Kwon, J. Um, J. Yeo, and H. Park, Phys. Rev. E 100, 052127 (2019).

[52] E. Aurell, R. Kawai, and K. Goyal, arXiv:1907.02671.

[53] D. J. Evans, E. G. D. Cohen, and G. P. Morriss, Phys. Rev. Lett. 71, 2401 (1993).

[54] G. Gallavotti and E. G. D. Cohen, Phys. Rev. Lett. 74, 2694 (1995).

[55] J. L. Lebowitz and H. Spohn, J. Stat. Phys. 95, 333 (1999).

[56] C. Maes, J. Stat. Phys. 95, 367 (1999).

[57] D. Andrieux, P. Gaspard, T. Monnai, and S. Tasaki, New J. Phys. 11, 043014 (2009).

[58] S. Chakravarty, Phys. Rev. Lett. 49, 681 (1982).

[59] A. J. Bray and M. A. Moore, Phys. Rev. Lett. 49, 1545 (1982).

[60] S. Chakravarty and A. J. Leggett, Phys. Rev. Lett. 52, 5 (1984).

[61] F. Guinea, V. Hakim, and A. Muramatsu, Phys. Rev. B 32, 4410 (1985).

[62] A. J. Leggett, S. Chakravarty, A. T. Dorsey, M. P. A. Fisher, A. Garg, and W. Zwerger, Rev. Mod. Phys. 59, 1 (1987).

[63] P. Cedraschi and M. Buttiker, Ann. Phys. (NY) 289, 1 (2001).

[64] K. L. Hur, Ann. Phys. (NY) 323, 2208 (2008).

[65] E. Aurell, R. Kawai, and K. Goyal, arXiv:1907.02671.

[66] R. Chetrite and K. Gaweędzki, Commun. Math. Phys. 282, 469 (2008).

[67] D. Segal, Phys. Rev. B 73, 205415 (2006).

[68] D. Segal, Phys. Rev. E 77, 021103 (2008).

[69] R. Sánchez, H. Thierschmann, and L. W. Molenkamp, New J. Phys. 19, 113040 (2017).

[70] D. Goury and R. Sánchez, Appl. Phys. Lett. 115, 092601 (2019).

[71] H. Touchette, Phys. Rep. 478, 1 (2009).
[72] W. Belzig and Y. V. Nazarov, Phys. Rev. Lett. 87, 197006 (2001).

[73] D. A. Bagrets and Y. V. Nazarov, Phys. Rev. B 67, 085316 (2003).

[74] C. Flindt, T. Novotný, and A.-P. Jauho, Europhys, Lett. 69, 475 (2005).

[75] F. Pistolesi, Phys. Rev. B 69, 245409 (2004).

[76] V. F. Maisi, D. Kambly, C. Flindt, and J. P. Pekola, Phys. Rev. Lett. 112, 036801 (2014).

[77] A. Komnik and A. O. Gogolin, Phys. Rev. Lett. 94, 216601 (2005).

[78] S. Pilgram, A. N. Jordan, E. V. Sukhorukov, and M. Büttiker, Phys. Rev. Lett. 90, 206801 (2003).

[79] C. Flindt, C. Fricke, F. Hohls, T. Novotný, K. Netočný, T. Brandes, and R. J. Haug, Proc. Natl. Acad. Sci. 106, 10116 (2009).

[80] Y. Utsumi, O. Entin-Wohlman, A. Ueda, and A. Aharony, Phys. Rev. B 87, 115407 (2013).

[81] M. Esposito, U. Harbola, and S. Mukamel, Phys. Rev. B 75, 155316 (2007).

[82] M. Esposito, U. Harbola, and S. Mukamel, Phys. Rev. E 76, 031132 (2007).

[83] M. Esposito, U. Harbola, and S. Mukamel, Rev. Mod. Phys. 81, 1665 (2009).

[84] T. Prosen, Phys. Rev. Lett. 107, 137201 (2011).

[85] T. Prosen, J. Phys. A: Math. Theor. 48, 373001 (2015).

[86] G. Gallavotti, Phys. Rev. Lett. 77, 4334 (1996).

[87] D. Andrieux and P. Gaspard, J. Chem. Phys. 121, 6167 (2004).

[88] D. Andrieux and P. Gaspard, J. Stat. Mech.: Theor. Exp. (2007) P02006.

[89] D. Segal and A. Nitzan, Phys. Rev. Lett. 94, 034301 (2005). 